\title{
GROUND STATES OF A BOSON QUANTUM LATTICE MODEL
}

\author{
Alexander E. Mazel ${ }^{1,2}$ and Yurii M. Suhov ${ }^{2,3,4}$
}

Abstract. A boson system on $\mathbb{Z}^{d}, d \geq 2$, is considered, with the Hamiltonian

$$
H=-1 / 2 \sum_{y} a_{y}^{+}(\Delta a)_{y}+\sum_{\left\langle y, y^{\prime}\right\rangle} U\left(N_{y}, N_{y^{\prime}}\right) .
$$

Here $a_{y}^{+}, a_{y}$ are the creation and annihilation operators, and $N_{y}=a_{y}^{+} a_{y}$. The nearestneighbor potential $U$ is given by: $U\left(n, n^{\prime}\right)=0$ if $n+n^{\prime} \leq m$ and $U\left(n, n^{\prime}\right)=+\infty$ if $n+n^{\prime}>m$ ( $m$ is a fixed positive integer). The Gibbs ensemble is determined by $\exp (-\beta(H-\mu N))$, where $N=\sum N_{y}$. By using a polymer expansion technique, we prove that, if $d \geq 2$ and $\mu>0$ and $\beta>0$ are large enough, the system has (precisely) two translation-periodic pure phases when $m$ is odd and a single pure phase when $m$ is even. We prove that the same is true of the ground states, and establish that the ground states are singular (degenerate) and correspond to 'dim' chess board configurations.

Key words and phrases: quantum lattice boson models, hard-core interaction, Gibbs states and measures, ground states, uniqueness and non-uniqueness, Feynman-Kac formula, classical and quantum contours, polymer expansion

\section{Introduction. The model and the main result}

In contrast to the theory of phase transitions in classical lattice systems, its quantum counterpart is much less developed. Few models have so far been studied at a rigorous level, and the results are not as complete as in classical cases. One of the major difficulties is that the analysis of the structure of the ground states - the starting point of any modern 'classical' work - is more difficult for a quantum model even

1 International Institute for the Earthquake Prediction Theory and Mathematical Geophysics, The Russian Academy of Sciences, Warshavskoe sh., 79-2, Moscow 113556, Russia

2 St John's College, Cambridge CB2 1TP and Isaac Newton Institute for Mathematical Sciences, Cambridge, CB3 0EH, England, UK

3 Statistical Laboratory, Department of Pure Mathematics and Mathematical Statistics, University of Cambridge, Cambridge CB2 1SB, England, UK

4 Institute for Problems of Information Transmission, The Russian Academy of Sciences, GSP-4 Moscow 101447, Russia

This research was supported in part by the Institute for Mathematics and its Applications, University of Minnesota, Minneapolis, MN 5545, USA 
when it is defined as an 'immediate' analogue of its classical prototype (which itself is often highly non-trivial). A striking example is the standard Heisenberg model (see [ FSiSp ] and [ DyLSi ]). Similar problems arise in dealing with the Hubbard models (see [ KL ], [ MeMir-So ], and the references therein).

This paper deals with a class of boson quantum lattice models with a (generalized) hard-core interaction. Some of these models were treated two decades ago by Ginibre [ G1 ] (a different method was used in [ R ]; Ginibre's method was improved, in some situations, in [ M2 ]). We use a technically different approach that allows us to extend Ginibre's result to a wider class of systems.

As usual, $\mathbb{Z}^{d}, d \geq 2$, denotes the cubic lattice in $\mathbb{R}^{d}$. The notation $V, V^{\prime}$, etc, is used below for a finite 'volume' (subset) of $\mathbb{Z}^{d}$. Given $V$, we denote by $\mathcal{H}_{V}$ the boson Fock-Hilbert space in $V$ and by $\mathcal{B}_{V}$ the $C^{*}$-algebra of bounded operators in $\mathcal{H}_{V}$. For $V^{\prime} \subset V, \mathcal{H}_{V}$ is identified with $\mathcal{H}_{V^{\prime}} \times \mathcal{H}_{V \backslash V^{\prime}}$. We use a standard orthonormal basis in $\mathcal{H}_{V}$ labeled by the (classical) configurations $X_{V}$ in $V$. That is, $X_{V}$ is a function $\mathbb{Z}^{d} \rightarrow \mathbb{Z}_{+}^{1}$, with $X(y)=0$ for $y$ outside $V$. The notation $X, Y$, etc., is used for configurations in $\mathbb{Z}^{d}$ (i.e. functions $\mathbb{Z}^{d} \rightarrow \mathbb{Z}_{+}^{1}$ ). Given a configuration $X$, we denote by $X_{V}$ the restriction of $X$ to $V .\left|X_{V}\right|$ denotes the number of particles in $X_{V}:\left|X_{V}\right|=\sum_{y \in V} X_{V}(y)$. By $a_{y}^{+}$and $a_{y}$ we denote, respectively, the boson creation and annihilation operators and by $N_{y}$ the particle-number operator $a_{y}^{+} a_{y}$, at site $y \in \mathbb{Z}^{d} . \quad N_{V}=\sum_{y \in V} N_{y}$ stands for the particle number operator in $V . \mathcal{B}$ denotes the inductive limit $\lim _{V>\mathbb{Z} d}$ ind $\mathcal{B}_{V}$ (the $C^{*}$-algebra of the quasilocal observables). The action of the space-translation group (e.g. on $\mathcal{B}$ ) is defined in a standard way. We refer to the property of translation-periodicity when we have translation-invariance for a sub-group of finite index. In particular, we talk about period two when the subgroup is of index two. The ergodicity (of a state of $\mathcal{B}$ ) is considered below with respect to the space-translation group; in a translation-periodic case it is with respect to the corresponding subgroup.

The Hamiltonian $H_{V}$ of the system in a finite volume $V$ reads as

$$
H_{V}=H_{V}^{0}+H_{V}^{\mathrm{int}}
$$

Here $H_{V}^{0}$ is the kinetic part

$$
H_{V}^{0}=-\frac{1}{d} \sum_{\left\langle y, y^{\prime}\right\rangle \in V} a_{y}^{+} a_{y}+\frac{1}{2 d} N_{V}
$$

the sum $\sum_{\left\langle y, y^{\prime}\right\rangle \in V}$ is over the (unordered) nearest-neighbor pairs $y, y^{\prime} \in V$. Observe that $H_{V}^{0}$ is nothing but the (second-quantized) discrete Laplacian in $V$, with Dirichlet's boundary conditions.

Furthermore, $H_{V}^{\mathrm{int}}$ is the potential part:

$$
H_{V}^{\mathrm{int}}=\sum_{\left\langle y, y^{\prime}\right\rangle \subset V} U\left(N_{y}, N_{y^{\prime}}\right) .
$$


Here, the nearest-neghbor potential $U$ prevents the accumulation of the particles:

$$
\begin{aligned}
U\left(n, n^{\prime}\right) & =0, & & \text { if } n+n^{\prime} \leq m, \\
& =+\infty, & & \text { otherwise }
\end{aligned}
$$

parameter $m$ is a fixed positive integer. Physically speaking, $U$ describes a kind of generalized hard-core repulsion (the case $m=1$ corresponds to a 'true' hard core and was considered by Ginibre [ G1 ]).

The models under consideration are natural quantum analogues of the classical models considered in [ $\mathrm{MaSu}$ ]. The classical model is obtained by omitting the kinetic part $H_{V}^{0}$ from Hamiltonian $H_{V}$.

Formally, the operator $H_{V}$ acts in the sub-space of $\mathcal{H}_{V}$ spanned by the basis vectors corresponding to admissible configurations $X_{V}$. A configuration $X_{V}$ (or $X$ ) is called admissible if $X_{V}(y)+X_{V}\left(y^{\prime}\right) \leq m\left(X(y)+X\left(y^{\prime}\right) \leq m\right)$ for any nearest-neighbor pair $\left\langle y, y^{\prime}\right\rangle \subset V\left(\left\langle y, y^{\prime}\right\rangle \subset \mathbb{Z}^{d}\right)$. In fact, we deal with the operators acting in a smaller space, $\mathcal{H}_{V}^{(Y)}$, generated by those configurations $X_{V}$ for which $X_{V}+Y_{V^{c}}$ is admissible. Here $Y$ is a fixed configuration (in $\mathbb{Z}^{d}$ ). This gives a kind of 'potential' boundary condition: the particles in $V$ are subject to the influence of a 'frozen' configuration $Y_{V^{c}}$. Of particular interest is the case where $Y$ coincides with one of the 'close-packed' admissible configurations $Y^{(l)}, l=0, \ldots, m$ :

$$
\begin{array}{lll}
Y^{(l)}(y)=l, & \text { if } \quad y \in \mathbb{Z}^{d} \quad \text { is even, } \\
Y^{(l)}(y)=m-l, & \text { if } \quad y \in \mathbb{Z}^{d} \quad \text { is odd. }
\end{array}
$$

Here and below we call $y=\left(y_{1}, \ldots, y_{d}\right)$ even (odd) if sum $\sum_{i} y_{i}$ is even (odd).

The Gibbs ensemble in $V$ is determined by a standard expression

$$
\exp \left(-\beta\left(H_{V}-\mu N_{V}\right)\right)
$$

where $\beta \geq 0$ is the inverse temperature and $\mu \in \mathbb{R}^{1}$ the chemical potential of the system. More precisely, we will deal with the Gibbs state $\phi_{V}^{(Y)}$ in $V$, with a boundary condition $Y$, which is given by

$$
\phi_{V}^{(Y)}(A)=\operatorname{tr}_{V} A \Pi_{V}^{(Y)}, \quad A \in \mathcal{B}_{V},
$$

where $\Theta_{V}^{(Y)}$ is the ortho-projection $\mathcal{H}_{V} \rightarrow \mathcal{H}_{V}^{(Y)}, \Pi_{V}^{(Y)}$ is the density matrix:

$$
\Pi_{V}^{(Y)}=\left(\Xi^{(Y)}(V)\right)^{-1} \Theta_{V}^{(Y)} \exp \left(-\beta\left(H_{V}^{(Y)}-\mu N_{V}\right)\right) \Theta_{V}^{(Y)},
$$

and $\operatorname{tr}_{V}$ stands for the trace in $\mathcal{H}_{V}$. The 'reduced' Hamiltonian $H_{V}^{(Y)}$ is defined by

$$
H_{V}^{(Y)}=\Theta_{V}^{(Y)} H_{V} \Theta_{V}^{(Y)}
$$

and $\Xi^{(Y)}(V)$ is the corresponding partition function:

$$
\Xi^{(Y)}(V)=\operatorname{tr}_{V} \Theta_{V}^{(Y)} \exp \left(-\beta\left(H_{V}^{(Y)}-\mu N_{V}\right)\right) \Theta_{V}^{(Y)}
$$


In the case $Y=Y^{(l)}$ we will use the simplified notations $\phi_{V}^{(l)}, \mathcal{H}_{V}^{(l)}, \Theta_{V}^{(l)}, \Xi^{(l)}(V)$, etc. Our goal is to study the $w^{*}$-limit

$$
\lim _{V \nearrow \mathbb{Z} d} \phi_{V}^{(Y)}
$$

of the Gibbs states $\phi_{V}^{(Y)}$. If the limit (1.11) exists, it gives a state of the $C^{*}$-algebra $\mathcal{B}$. A standard low-density (or high-temperature) expansion method (see, e.g. [ BR ], Vol 2, Chap. 3.2.5) makes it possible to prove that there exists $\mu_{0}=\mu_{0}(d, m) \in \mathbb{R}^{1}$ such that for $\mu \leq \mu_{0}$ and any $\beta>0$ the limit (1.11) exists and the limiting state $\phi$ does not depend on the boundary condition. In fact, one can prove a somewhat stronger assertion that the KMS-state for the corresponding derivation of $\mathcal{B}$ (see (1.13)) is unique provided that the condition $\mu \leq \mu_{0}$ is fulfilled. In this paper we consider an opposite situation where $\mu$ is large. The result is the following Theorem.

Theorem 1. Let dimension $d \geq 2$ and positive integer $m$ be fixed. There exist $\bar{\mu}=\bar{\mu}(d)>0$ and $\bar{\beta}=\bar{\beta}(d)>0$, such that, for any pair $(\beta, \mu)$ with $\mu \geq \mu^{(0)}=\bar{\mu} m$ and $\beta \geq \beta^{(0)}=\bar{\beta}, m$, the following assertions hold.

(i) If $m$ is even, the limit (1.11) exists for any periodic boundary condition $Y$. The limiting state $\phi^{0}$ does not depend on $Y$ and is translation-invariant.

(ii) If $m$ is odd, there exist exactly two different translation-periodic, ergodic limiting states (1.11), $\phi^{+}$and $\phi^{-}$. More precisely,

$$
\phi^{+}=\lim _{V \nearrow \mathbb{Z}^{d}} \phi_{V}^{(l)} \quad \text { for } \quad l=\frac{1}{2}(m+1), \ldots, m,
$$

and

$$
\phi^{-}=\lim _{V \nearrow_{\mathbb{Z} d}} \phi_{V}^{(l)} \quad \text { for } \quad l=0, \ldots, \frac{1}{2}(m-1),
$$

and the limit (1.11) coincides, for any translation-periodic $Y$, with a mixture of $\phi^{+}$ and $\phi^{-}$. States $\phi^{ \pm}$are transformed into each other by the unit space-shifts; they are translation-periodic with period two and ergodic.

To explain the result contained in Theorem 1 , observe that, for $\mu>0$, the classical system, with potential $U$, has the translation-periodic ground states that are precisely the close-packed configurations $Y^{(l)}$. The classical analogue of Theorem 1 (see $[\mathrm{MaSu}]$ ) states that, among the classical ground states, those that generate translationperiodic thermodynamical phases are precisely $Y^{(m / 2)}$ for $m$ even and $Y^{(m / 2 \pm 1 / 2)}$ for $m$ odd. The point is that these ground states are 'dominant', in the sense that they have greater entropy of low-energy excitations. The authors showed in [ MaSu ] that there exist a bound $(\beta \mu)^{0}$, uniform in $m$, such that, for $\beta \mu \geq(\beta \mu)^{0}$, only the dominant ground states generate the translation-periodic Gibbs measures associated with $H_{V}^{\mathrm{int}}$.

In the quantum case, the nature of the dominance is slightly changed: the main factor extracting the dominant states is the entropy of 'jumps' related to the kinetic part of the Hamiltonian. Because of that difference between the classical and quantum cases, we fail to obtain a bound for $\beta^{0}$ and $\mu^{0}$, which is uniform in $m$.

It is possible to prove (we do not do so in this paper) that the limiting states mentioned in Theorem 1 satisfy the KMS boundary condition, with respect to the derivation

$$
\delta(A)=\lim _{V \nearrow \mathbb{Z} d} i\left[H_{V}-\mu N_{V}, A\right]
$$


[More precisely, we have to modify slightly the standard definition of a KMS-state (see [ BR ], Vol 2, Chap. 5.3), because of the hard-core condition.] However, it is still an open question that the states indicated in Theorem 1 are the unique translation-periodic KMS states of the system.

The proof of Theorem 1 is carried in Sections 2-4. In Section 2 we introduce, by using the Feynman-Kac (FK) formula, a geometrical representation of the models under consideration, in terms of quantum contours (which are quantum analogues of the classical contours (or cylinders, in the terminology used in [ $\mathrm{MaSu}]$ )). In Section 3 we develop a specific scheme of polymer expansion, and in Section 4 complete the proof.

We should note that the FK formula (in various versions) was used, to study quantum models, for a long time and by many authors. We refer the reader to papers [ G1 ], [ G2 ], [ M2 ], [ P ], [ Su ], [ OPiSu ] and book [ BR ] (see also the references therein).

Our second result concerns the quantum ground states, defined here as the limits of the Gibbs states as $\beta \rightarrow \infty$. We say that a state $\psi$ is classical if there exists a probability measure $\Psi$, on the space of the admissible configurations $X$ in $\mathbb{Z}^{d}$, such that, for any finite $V \subset \mathbb{Z}^{d}$ and any $A \in \mathcal{B}_{V}$,

$$
\psi(A)=\int \Psi(d X) f_{A}(X)
$$

where function $f$ is given by the diagonal matrix elements of $A$ in our standard basis in $\mathcal{H}_{V}: f_{A}(X)=A\left(X_{V}, X_{V}\right)$. We call a classical state $\psi$ singular if measure $\Psi$ is concentrated at a single configuration.

Theorem 2. For any $\mu>\mu^{(0)}=\bar{\mu} m$ (with constant $\bar{\mu}$ from Theorem 1) there exist the $w^{*}$-limits of the states $\phi^{0}$ and $\phi^{ \pm}$constructed in Theorem 1:

$$
\bar{\phi}^{0}=\lim _{\beta \rightarrow \infty} \phi^{0}, \quad \bar{\phi}^{ \pm}=\lim _{\beta \rightarrow \infty} \phi^{ \pm}
$$

which are singular classical states corresponding to the configurations $Y^{(l)}$ with $l=$ $m / 2$ and $l=(m \pm 1) / 2$, respectively.

A well-known definition of a ground state refers to a positive spectrum condition, for the generator of the unitary group induced, in the GNS-representation, by derivation (1.13). See, e.g. [ BR ], Vol 2, Sect 5.3.3. The classical singular states corresponding to the close-packed configurations $Y^{(l)}, l=0, \ldots, m$, and in particular, the states indicated in Theorem 2, obviously satisfy this condition (provided that $\mu$ is large enough). Again, there is an open question that these states are the only pure translation-periodic states with that property. Furthermore, it is interesting to clarify what additional conditions are needed to extract, from the whole family of classical singular close-packed states, those mentioned in Theorem 2.

The proof of Theorem 2 is obtained as a by-product, in the course of proving Theorem 1.

We conclude this section by noting that the convergence in $(1.12 \mathrm{a}, \mathrm{b})$ and $(1.15)$ is equivalent to the convergence of the matrix elements of the reduced density matrices. In the case of $(1.12 \mathrm{a}, \mathrm{b})$ we have to check that, for any fixed finite $V^{0} \subset \mathbb{Z}^{d}$, and any pair of admissible configurations $X_{V^{0}}, X_{V^{0}}^{\prime}$ in $V^{0}$, the limits

$$
\lim _{V \nearrow_{\mathbb{Z}^{d}}} \pi_{V, V^{\mathrm{o}}}^{(l)}\left(X_{V^{\mathrm{o}}}, X_{V^{\mathrm{o}}}^{\prime}\right)=\pi_{V^{\mathrm{o}}}^{(l)}\left(X_{V^{\mathrm{o}}}, X_{V^{\mathrm{o}}}^{\prime}\right)
$$


exist and give the corresponding states. Here, $\pi_{V, V^{0}}^{(l)}\left(X_{V^{0}}, X_{V^{0}}^{\prime}\right)$ stands for the matrix element of the reduced density matrix $\pi_{V, V^{0}}^{(l)}$ that is related to the density matrix $\Pi_{V}$ (see (1.8)) by

$$
\pi_{V, V^{0}}^{(l)}=\operatorname{tr}_{V \backslash V^{\mathrm{o}}} \Pi_{V}
$$

The translation-invariance and translation-periodicity properties of a limiting state are equivalent to the corresponding properties of the family $\left\{\pi_{V^{0}}\right\}$ of the limiting reduced matrices. The ergodicity follows from a correlation-decay property for the matrix elements $\left\{\pi_{V^{\mathrm{o}}}^{\cdot}\left(X_{V^{\mathrm{o}}}, X_{V^{\mathrm{o}}}^{\prime}\right)\right\}$. See again [BR ], vol 1 .

In the case of (1.15) we have to check that the limiting reduced density matrices $\pi^{\cdot}$ obey

$$
\begin{aligned}
\lim _{\beta \rightarrow \infty} \pi_{V^{0}}^{0}\left(X_{V^{0}}, X_{V^{0}}^{\prime}\right) & =1, \quad \text { if } X_{V^{0}}=X_{V^{0}}^{\prime}=Y_{V^{0}}^{(m / 2)} \\
& =0, \quad \text { otherwise }
\end{aligned}
$$

and

$$
\begin{aligned}
\lim _{\beta \rightarrow \infty} \pi_{V^{0}}^{ \pm}\left(X_{V^{0}}, X_{V^{0}}^{\prime}\right) & =1, \quad \text { if } X_{V^{0}}=X_{V^{0}}^{\prime}=Y_{V^{0}}^{(m / 2 \pm 1 / 2)} \\
& =0, \quad \text { otherwise. }
\end{aligned}
$$

\section{Some technical tools}

The Feynman-Kac formula. As mentioned in Section 1, one of the main tools used for proving Theorems 1 and 2 is the FK-formula. In a standard form, it may be found, e.g. in [ Gi2 ] or [ BR ], vol 2, Sec. 3.3.2 and 3.3.3. We use a version of the FK-formula based on the concept of a quantum configuration. More precisely, we consider sequences $\mathbf{X}_{V}=\left(X_{V}^{0}, X_{V}^{1}, \ldots, X_{V}^{n}\right)$ of admissible classical configurations in $V$, with $X_{V}^{s}$ and $X_{V}^{s+1}$ differing at two nearest-neighbor lattice sites only, $y_{s}^{ \pm}$, with $X_{V}^{s+1}\left(y_{s}^{ \pm}\right)-X_{V}^{s}\left(y_{s}^{ \pm}\right)= \pm 1, s=0, \ldots, n-1$. Such families are called quantum configurations in $V$.

Lemma 2.1. For any $\beta, \mu$ and $V$, any pair of configurations $X_{V}, X_{V}^{\prime}$ in $V$, and any configuration $Y$, the matrix element $E_{V}^{(Y)}\left(X_{V}, X_{V}^{\prime}\right)$ of the operator $\Theta_{V}^{(Y)} \exp \left(-\beta\left(H_{V}^{(Y)}-\mu N_{V}\right)\right) \Theta_{V}^{(Y)}$ is given by

$$
\begin{gathered}
E_{V}^{(Y)}\left(X_{V}, X_{V}^{\prime}\right)=\exp \left(\beta \mu\left|\mathbf{X}_{V}\right|-\beta\left|\mathbf{X}_{V}\right|\right) \sum_{n=0}^{\infty} \frac{1}{n !}\left(\frac{\beta}{2 d}\right)^{n} \\
\sum_{V}^{n-1} X_{V}^{i}\left(y_{i}^{-}\right) \\
\mathbf{X}_{V}=\left(X_{V}^{0}, X_{V}^{1}, \ldots, X_{V}^{n}\right): i=0 \\
X_{V}^{0}=X_{V}, X_{V}^{n}=X_{V}^{\prime} \\
X_{V}^{s}+Y_{V^{c}} \text { is admissible } \\
=0, \quad \text { otherwise. }
\end{gathered}
$$

Here, $\left|\mathbf{X}_{V}\right|$ is the number of particles in (any of) classical configurations $X_{V}^{s}$ representing $\mathbf{X}_{V}$. 
We omit the proof of Lemma 2.1: it is of standard probabilistic character and may be immediately deduced from the 'usual' FK formula (see the above references).

By using Lemma 2.1, we obtain the following formulas for the partition function $\Xi^{(Y)}(V)$ and the matrix elements of the reduced density matrix $\pi_{V, V^{0}}^{(Y)}$ :

$$
\Xi^{(Y)}(V)=\sum_{X_{V}} E_{V}^{(Y)}\left(X_{V}, X_{V}\right)
$$

and for any admissible configurations $X_{V^{0}}, X_{V^{\text {o }}}^{\prime}$ in $V^{0}$,

$$
\pi_{V, V^{0}}^{(Y)}\left(X_{V^{0}}, X_{V^{0}}^{\prime}\right)=\frac{\Xi^{(Y)}\left(V, V^{0} ; X_{V^{0}}, X_{V^{0}}^{\prime}\right)}{\Xi^{(Y)}(V)} .
$$

Here,

$$
\Xi^{(Y)}\left(V, V^{0} ; X_{V^{0}}, X_{V^{0}}^{\prime}\right)=\sum_{X_{V \backslash V^{0}}} E_{V}^{(Y)}\left(X_{V^{0}}+X_{V \backslash V^{0}}, X_{V^{0}}^{\prime}+X_{V \backslash V^{0}}\right) .
$$

The sum in (2.2) is over the configurations $X_{V}$ such that $X_{V}+Y_{V^{c}}$ is admissible, and in (2.4) over the configurations $X_{V \backslash V^{0}}$ such that $X_{V^{0}}+X_{V \backslash V^{0}}+Y_{V^{c}}$ and $X_{V^{0}}^{\prime}+X_{V \backslash V^{0}}+Y_{V^{c}}$ are admissible.

Lemma 2.1 and formulas $(2.2)-(2.4)$ allow us to treat the quantum model under consideration as a system of 'classical' statistical mechanics, where one deals with quantum configurations (and later with quantum contours). The statistical weight, $w\left(\mathbf{X}_{V}\right)$, of a quantum configuration $\mathbf{X}_{V}=\left(X_{V}^{0}, \ldots, X_{V}^{n}\right)$ (where each $X_{V}^{s}+Y_{V^{c}}$ is admissible) is given by

$$
w\left(\mathbf{X}_{V}\right)=\exp \left(\beta(\mu-1)\left|\mathbf{X}_{V}\right|-\beta(\mu-1)\left|Y_{V}\right|\right)\left(\frac{\beta}{2 d}\right)^{n} \frac{1}{n !} \prod_{i=0}^{n-1} X_{V}^{i}\left(y_{i}^{-}\right)
$$

and the partition function (2.2) may be rewritten as

$$
\Xi^{(Y)}(V)=\exp \left(\beta(\mu-1)\left|Y_{V}\right|\right) \sum_{\substack{\mathbf{X}_{V}: X_{V}^{0}=X_{V}^{n}, X_{V}^{s}+Y_{V^{c}} \text { is admissible }}} w\left(\mathbf{X}_{V}\right) .
$$

Theorems 1 and 2 may therefore be re-formulated as statements about the structure of the set of the Gibbs (or DLR) measures for an ensemble of quantum configurations. Furthermore, the properties of the ensemble of quantum configurations may be expressed in terms of an appropriate ensemble of quantum contours.

Henceforth, we depart from the original quantum model and concentrate on the analysis of the corresponding ensembles of quantum configurations, with statistical weight (2.5). We focus attention on studying of cyclic quantum configurations $\mathbf{X}_{V}$ (with $X_{V}^{0}=X_{V}^{n}$ ) figuring in (2.6). The extension of the argument to the general case needed for (2.4) leads to a 'local' perturbation of the space of quantum configurations, which does not change its phase diagram; the corresponding technical details are omitted from the paper. 
Geometry of the classical configurations. Our main goal in Section 2 is to introduce the concept of a quantum contour which will then form a base for an appropriate polymer expansion. The definition and properties of quantum contours are related to the definition and properties of classical contours (or briefly, contours). A contour $\gamma$ is a triple $(\widetilde{\gamma}, E, I)$, where

(i) $\tilde{\gamma}=\tilde{\gamma}(\gamma)$ is a finite connected set of the plaquettes of the dual lattice $\widetilde{\mathbb{Z}}^{d}=$ $\left\{y+\left(\frac{1}{2}, \ldots, \frac{1}{2}\right): y \in \mathbb{Z}^{d}\right\}$ such that

(i.1) either 0 or 2 or 4 plaquettes from $\tilde{\gamma}$ meet at any $(d-2)$-dimensional face of $\widetilde{\mathbb{Z}}^{d}$,

(i.2) if precisely two plaquettes from $\tilde{\gamma}$ meet at a given $(d-2)$-dimensional face of $\widetilde{\mathbb{Z}}^{d}$, then they are not parallel;

(ii) $E=E(\gamma)$ and $I=I(\gamma)$ are two integers from $\{0,1, \ldots, m\}$ such that

(ii.1) $I>E$ for $\tilde{\gamma}$ even,

(ii.2) $I<E$ for $\tilde{\gamma}$ odd.

Set $\tilde{\gamma}=\tilde{\gamma}(\gamma)$ figuring in (i) is called a (classical) interface. We call an interface $\tilde{\gamma}$ even (odd) when there exists an odd (even) $x \in \mathbb{Z}^{d}$ such that

(a) $x$ is adjacent to some of the plaquettes from $\tilde{\gamma}$;

(b) for any natural $n$ there exists a self-avoiding path of length $n$, along the bonds of $\mathbb{Z}^{d}$, such that it does not intersect the plaquettes from $\tilde{\gamma}$.

It is not hard to see that every interface $\tilde{\gamma}$ separates a finite connected and simple-connected subset of $\mathbb{Z}^{d}$, called the interior of $\tilde{\gamma}$ and denoted by Int $\bar{\gamma}$, from its complement called the exterior of $\tilde{\gamma}$ and denoted by Ext $\tilde{\gamma}$. In the case where $\tilde{\gamma}$ is considered as the component of a contour $\gamma$, we speak about the interior and exterior of a contour and use the notation Int $(\gamma)$.

Two contours, $\gamma^{\prime}=\left(\tilde{\gamma}^{\prime}, E^{\prime}, I^{\prime}\right)$ and $\gamma^{\prime \prime}=\left(\widetilde{\gamma}^{\prime \prime}, E^{\prime \prime}, I^{\prime \prime}\right)$, are called compatible if one of the following conditions holds

(i) $\tilde{\gamma}^{\prime} \cap \widetilde{\gamma}^{\prime \prime}=\emptyset$, Int $\tilde{\gamma}^{\prime} \subset$ Int $\tilde{\gamma}^{\prime \prime}$ and $I^{\prime \prime}=E^{\prime}$;

(ii) $\tilde{\gamma}^{\prime} \cap \widetilde{\gamma}^{\prime \prime}=\emptyset$, Int $\widetilde{\gamma}^{\prime \prime} \subset$ Int $\tilde{\gamma}^{\prime}$ and $I^{\prime}=E^{\prime \prime}$;

(iii) $\widetilde{\gamma}^{\prime} \cap \widetilde{\gamma}^{\prime \prime}=\emptyset$, Int $\widetilde{\gamma}^{\prime} \cap$ Int $\widetilde{\gamma}^{\prime \prime}=\emptyset$ and $E^{\prime \prime}=E^{\prime}$;

(iv) $\tilde{\gamma}^{\prime} \cap \widetilde{\gamma}^{\prime \prime} \neq \emptyset$, Int $\tilde{\gamma}^{\prime} \subset$ Int $\tilde{\gamma}^{\prime \prime}$ and either $E^{\prime \prime}>I^{\prime \prime}=E^{\prime}>I^{\prime}$ or $E^{\prime \prime}<I^{\prime \prime}=$ $E^{\prime}<I^{\prime}$

(v) $\tilde{\gamma}^{\prime} \cap \widetilde{\gamma}^{\prime \prime} \neq \emptyset$, Int $\widetilde{\gamma}^{\prime \prime} \subset$ Int $\bar{\gamma}^{\prime}$ and either $E^{\prime}>I^{\prime}=E^{\prime \prime}>I^{\prime \prime}$ or $E^{\prime}<I^{\prime}=$ $E^{\prime \prime}<I^{\prime \prime}$

(vi) $\tilde{\gamma}^{\prime} \cap \tilde{\gamma}^{\prime \prime} \neq \emptyset$, Int $\tilde{\gamma}^{\prime} \cap$ Int $\tilde{\gamma}^{\prime \prime}=\emptyset$ and either $I^{\prime \prime}>E^{\prime \prime}=E^{\prime}>I^{\prime}$ or $I^{\prime \prime}<E^{\prime \prime}=E^{\prime}<I^{\prime}$.

A finite collection $\left\{\boldsymbol{\gamma}_{i}\right\}$ of contours is compatible if any two contours from the collection not separated by any third contour are compatible. [We say that a contour $\gamma$ separates contours $\gamma^{\prime}$ and $\gamma^{\prime \prime}$ if the corresponding interfaces $\tilde{\gamma}, \tilde{\gamma}^{\prime}$ and $\tilde{\gamma}^{\prime \prime}$ obey Int $\tilde{\gamma}^{\prime} \subset$ Int $\tilde{\gamma}$ and Int $\tilde{\gamma}^{\prime \prime} \subset$ Ext $\tilde{\gamma}$ or Int $\tilde{\gamma}^{\prime \prime} \subset$ Int $\tilde{\gamma}$ and Int $\tilde{\gamma}^{\prime} \subset$ Ext $\tilde{\gamma}$.] Note that for any finite compatible collection $\left\{\boldsymbol{\gamma}_{i}\right\}$ all external contours have the same value $E$ which we denote by $E\left(\left\{\gamma_{i}\right\}\right)$. Here and below, we call a contour $\gamma$ external (in a given collection) if Int $\tilde{\gamma}$ is not contained in the interior of any other contour from the collection.

An important fact is that there exists a 1-1 correspondence between the admissible configurations $X$ coinciding with some $Y^{(l)}$ outside a finite subset of $\mathbb{Z}^{d}$ and the finite compatible collections of contours $\left\{\boldsymbol{\gamma}_{i}\right\}$ with $E\left(\left\{\boldsymbol{\gamma}_{i}\right\}\right)=l$. To describe this 
correspondence, it is convenient to equip the set of the contours $\gamma$ with a partial order determined by the set-theoretical inclusion of the interiors Int $\gamma$. Given $y \in \mathbb{Z}^{d}$ and a compatible collection of contours $\left\{\boldsymbol{\gamma}_{i}\right\}$, consider the contours from $\left\{\boldsymbol{\gamma}_{i}\right\}$, with the interors containing $y$, and select, from among them a minimal one, in the sense of the above order. Denote the minimal contour by $\gamma(y)=(\widetilde{\gamma}(y), E(y), I(y))$, if it exists, and write $\gamma(y)=\emptyset$, otherwise. If $\gamma(y) \neq \emptyset$, then the admissible configuration $X$ corresponding to $\left\{\boldsymbol{\gamma}_{i}\right\}$ has the value $X(y)=I(y)$. On the other hand, if $\gamma(y)=\emptyset$ then $X(y)=E\left(\left\{\boldsymbol{\gamma}_{i}\right\}\right)$.

The correspondence just introduced is especially useful because of the following identity:

$$
\left|Y_{V}^{(l)}\right|-\left|X_{V}\right|=\frac{1}{2 d} \sum_{i}\left|\widetilde{\gamma}_{i}\right| \cdot\left|E\left(\gamma_{i}\right)-I\left(\gamma_{i}\right)\right|
$$

where $V$ is an (arbitrary) finite volume such that $X_{V^{c}}=Y_{V^{c}}^{(l)}$. Here, and below $\left|\widetilde{\gamma}_{i}\right|$ denotes the number of plaquettes in $\tilde{\gamma}_{i}$.

Geometry of quantum configurations. In this sub-section we construct, for quantum configurations, an analogue of contour representation. From now on we are working with cyclic quantum configurations. Recall that in a cyclic quantum configuration $\mathbf{X}_{V}=\left(X_{V}^{0}, \ldots, X_{V}^{n}\right), n=n\left(\mathbf{X}_{V}\right)$ is always even, and $X_{V}^{0}=X_{V}^{n}$. In the special case $n\left(\mathbf{X}_{V}\right)=0$, the cyclic quantum configuration consists of a single classical configuration $X_{V}$. We assume that, in a given quantum configuration $\mathrm{X}_{V}=\left(X_{V}^{0}, \ldots, X_{V}^{n}\right)$, in $V$, the classical configurations $X_{V}^{i}+Y_{V^{c}}^{(l)}$ are admissible for some $l=0, \ldots, m$, and write $X^{i}=X_{V}^{i}+Y_{V^{c}}^{(l)}, \quad i=1, \ldots, n$. Furthermore, we write $\mathbf{X}=\left(X^{0}, \ldots, X^{n}\right)$ and call $\mathbf{X}$ a quantum configuration in $\mathbb{Z}^{d}$. As was pointed out before, each classical configuration $X^{i}$ corresponds to a unique compatible collection of contours $\left\{\boldsymbol{\gamma}_{j}^{i}\right\}$, with $E\left(\left\{\gamma_{j}^{i}\right\}\right)=l$. Consider the union $\cup_{i, j} \widetilde{\gamma}_{j}^{i}$ of the interfaces from all configurations $X^{i}, i=1, \ldots, n$, (briefly, the interfaces from $\mathbf{X}$ ). This is a finite set of plaquettes of $\widetilde{\mathbb{Z}}^{d}$, and it can be decomposed into the connected components. Let $K=K(\mathrm{X})$ be the number of these components. We index them by a superscript $k$ taking values $1, \ldots, K$ (in an arbitrary order). Next, we partition the collection of the contours $\gamma_{j}^{i}$, from all configurations $X^{i}, i=1, \ldots, n$ (briefly, the collection of the contours from $\mathrm{X}$ ) into sub-collections, $\left\{\boldsymbol{\gamma}_{t}^{(k)}\right\}, k=1, \ldots, K$, in a such a way that the interfaces of the contours $\gamma_{t}^{(k)}$, with a fixed $k$, are exactly the interfaces from the $k$-th connected component of $\cup_{i, j} \widetilde{\gamma}_{j}^{i}$. A single sub-collection $\left\{\boldsymbol{\gamma}_{t}^{(k)}\right\}$ is called a quantum interface. This procedure leads us further to the concept of a quantum contour.

A quantum contour $\zeta$ is a finite family of classical contours $\left\{\gamma_{j}^{i}, i=0,1, \ldots\right.$, $\left.n(\boldsymbol{\zeta}) ; j=1,2, \ldots, n_{i}(\boldsymbol{\zeta})\right\}$ such that

(i) for any fixed $i=0,1, \ldots, n(\boldsymbol{\zeta})$, the subcollection $\left\{\boldsymbol{\gamma}_{j}^{i}: j=1, \ldots, n_{i}(\boldsymbol{\zeta})\right\}$ is a compatible collection of contours with $E\left(\left\{\gamma_{j}^{i}\right\}\right)=l$, which defines an admissible classical configuration $X^{i}=X^{i}(\zeta)$ coinciding with $Y^{(l)}$ outside some finite $V \subset \mathbb{Z}^{d}$

(ii) the collection $\mathbf{X}_{V}(\zeta)=\left(X_{V}^{0}, \ldots, X_{V}^{n(\boldsymbol{\zeta})}\right)$ is a cyclic quantum configuration in $V$ (or equivalently, $\mathbf{X}(\boldsymbol{\zeta})=\left(X^{0}, \ldots, X^{n(\zeta)}\right)$ is a cyclic quantum configuration in $\left.\mathbb{Z}^{d}\right)$; 
(iii) the union $\tilde{\zeta}=\cup_{i, j} \tilde{\gamma}_{j}^{i}(\zeta)$ of the interfaces $\tilde{\gamma}_{j}^{i}(\zeta)$ of all contours from $\zeta$ is a connected set of plaquettes of $\widetilde{\mathbb{Z}}^{d}$.

Observe that $\tilde{\zeta}=\widetilde{\zeta}(\zeta)$ is a unique quantum interface in quantum configuration $\mathbf{X}_{V}(\boldsymbol{\zeta})$ (or $\mathbf{X}(\boldsymbol{\zeta})$ ). Also note that the quantum configuration $\mathbf{X}(\boldsymbol{\zeta})$ is uniquely determined by $\zeta$.

A collection $\left\{\boldsymbol{\zeta}_{r}\right\}$ of quantum contours is compatible if the quantum interfaces $\widetilde{\zeta}\left(\zeta_{r}\right)$ do not intersect each other for different $r$ 's and $\left\{\boldsymbol{\gamma}_{j}^{0}\left(\boldsymbol{\zeta}_{r}\right): j=1, \ldots, n_{0}\left(\boldsymbol{\zeta}_{r}\right) ; r=\right.$ $0,1, \ldots\}$ is a compatible collection of classical contours.

The construction described before allows us to assign, to any quantum configuration $\mathbf{X}$ in $\mathbb{Z}^{d}$, a unique compatible collection of quantum contours (by decomposing the union $\cup_{i, j} \widetilde{\gamma}_{j}\left(X^{i}(\mathbf{X})\right)$ into the connected components). However, unlike the compatible collections of classical contours, a finite compatible collection of quantum contours defines, in general, not a single quantum configuration in $\mathbb{Z}^{d}$ but an entire family of them. To explain this fact, note that any quantum configuration describes nothing more than the sequence of particle jumps. A quantum contour $\zeta$ (that has a unique corresponding quantum configuration $\mathbf{X}(\boldsymbol{\zeta}))$ is a sequence of $n(\boldsymbol{\zeta})$ jumps. Now observe that, for any compatible collection of quantum contours, the jumps corresponding to distinct quantum contours are independent. Hence, with a given compatible collection of quantum contours $\left\{\boldsymbol{\zeta}_{r}\right\}$ one can associate precisely

$$
\frac{\left(\sum_{r} n\left(\boldsymbol{\zeta}_{r}\right)\right) !}{\prod_{r} n\left(\zeta_{r}\right) !}
$$

quantum configurations that correspond to various possibilities to intermit the jumps from different $\mathbf{X}\left(\boldsymbol{\zeta}_{r}\right)$ 's with each other.

Formally, the procedure is as follows. Fix a compatible collection $\left\{\boldsymbol{\zeta}_{r}\right\}$ of quantum contours. For each $i$ and $r$ we have a family of classical contours $\left\{\boldsymbol{\gamma}_{j}^{i}\left(\boldsymbol{\zeta}_{r}\right): j=1, \ldots, n_{i}\left(\boldsymbol{\zeta}_{r}\right)\right\}$ which determines a (unique) admissible configuration $X^{i}\left(\mathbf{X}\left(\zeta_{r}\right)\right)$. Re-index the pairs $(i, r)$ by a single label, $s$, taking values $0, \ldots, n\left(\left\{\boldsymbol{\zeta}_{r}\right\}\right), n\left(\left\{\boldsymbol{\zeta}_{r}\right\}\right)=\sum_{r} n\left(\boldsymbol{\zeta}_{r}\right)$, so that for any $\left(i_{1}, r\right), \quad\left(i_{2}, r\right)$ with $i_{1}<i_{2}$, the corresponding labels $s_{1}, s_{2}$ obey $s_{1}<s_{2}$. It is not hard to check that the number of different labelings with this property is given by (2.7). Given such a labeling, we construct the (unique) corresponding quantum configuration $\mathbf{X}\left(\left\{\boldsymbol{\zeta}_{r}\right\}\right)=$ $\left(X^{0}\left(\left\{\boldsymbol{\zeta}_{r}\right\}\right), \ldots, X^{n\left(\left\{\boldsymbol{\zeta}_{r}\right\}\right)}\left(\left\{\boldsymbol{\zeta}_{r}\right\}\right)\right)$ inductively, as follows.

(a) Configuration $X^{0}\left(\left\{\boldsymbol{\zeta}_{r}\right\}\right)$ is determined by the collection of classical contours $\left\{\gamma_{j}^{0}\left(\zeta_{r}\right): j=1, \ldots, n_{0}\left(\zeta_{r}\right) ; r=0,1, \ldots\right\}$.

(b) Suppose that configuration $X^{s}\left(\left\{\boldsymbol{\zeta}_{r}\right\}\right)$ is already determined and take the pair $\left(i_{0}, r_{0}\right)$ labeled by value $s+1$. We construct the configuration $X^{s+1}\left(\left\{\boldsymbol{\zeta}_{r}\right\}\right)$ by replacing, in compatible collection of contours corresponding to $X^{s}\left(\left\{\boldsymbol{\zeta}_{r}\right\}\right)$, the sub-collection $\left\{\boldsymbol{\gamma}_{j}^{i_{0}-1}\left(\boldsymbol{\zeta}_{r_{0}}\right): j=1, \ldots, n_{i_{0}-1}\left(\boldsymbol{\zeta}_{r_{0}}\right)\right\}$ by $\left\{\boldsymbol{\gamma}_{j}^{i_{0}}\left(\boldsymbol{\zeta}_{r_{0}}\right): j=\right.$ $\left.1, \ldots, n_{i_{0}}\left(\zeta_{r_{0}}\right)\right\}$.

Now assign, to a compatible collection of quantum contours $\left\{\boldsymbol{\zeta}_{r}\right\}$, the statistical weight

$$
w\left(\left\{\boldsymbol{\zeta}_{r}\right\}\right)=\sum_{\mathbf{X}\left(\left\{\boldsymbol{\zeta}_{r}\right\}\right)} w\left(\mathbf{X}\left(\left\{\boldsymbol{\zeta}_{r}\right\}\right)\right)
$$


where the sum is extended to all quantum configurations $\mathbf{X}\left(\left\{\boldsymbol{\zeta}_{r}\right\}\right)$ associated with $\left\{\boldsymbol{\zeta}_{r}\right\}$. [Formally, statistical weight $(2.5)$ is assigned only to the quantum configurations in a finite volume. But it is easy to check that there exists a unique $l$ such that for any $s$ the configuration $X^{s}\left(\mathbf{X}\left(\left\{\boldsymbol{\zeta}_{r}\right\}\right)\right)$ coincides with $Y^{(l)}$ outside some finite $V$. Substituting this $V$ and $Y=Y^{(l)}$ into (2.5), we get a correct expression for $w\left(\mathbf{X}\left(\left\{\boldsymbol{\zeta}_{r}\right\}\right)\right)$.] Obviously, the statistical weight is the same for each quantum configuration $\mathbf{X}\left(\left\{\boldsymbol{\zeta}_{r}\right\}\right)$ associated with $\left\{\boldsymbol{\zeta}_{r}\right\}$. Moreover,

$$
\frac{\left(\sum_{r} n\left(\boldsymbol{\zeta}_{r}\right)\right) !}{\prod_{r} n\left(\boldsymbol{\zeta}_{r}\right) !} w\left(\mathbf{X}\left(\left\{\boldsymbol{\zeta}_{r}\right\}\right)\right)=\prod_{r} w\left(\boldsymbol{\zeta}_{r}\right)
$$

where, for an arbitrary quantum contour $\boldsymbol{\zeta}$,

$$
w(\boldsymbol{\zeta})=w(\mathbf{X}(\boldsymbol{\zeta}))
$$

and $w(\mathbf{X}(\boldsymbol{\zeta}))$ is calculated by means of (2.5), with the corresponding $V$ and $Y=Y^{(l)}$. Now, for any $l$, expression $(2.6)$ can be re-written as

$$
\begin{aligned}
& \Xi^{(l)}(V)=\exp \left(\beta(\mu-1)\left|Y_{V}^{(l)}\right|\right) \quad \sum_{\widetilde{\zeta}} \prod_{r} w\left(\zeta_{r}\right) . \\
& \left\{\boldsymbol{\zeta}_{r}\right\}:\left\{\widetilde{\zeta}_{r}\right\} \subset V, \\
& X_{V}^{s}\left(\mathbf{X}\left(\left\{\boldsymbol{\zeta}_{r}\right\}\right)\right)+Y_{V^{c}}^{(l)} \\
& \text { is admissible }
\end{aligned}
$$

The external factor $\exp \left(\beta(\mu-1)\left|Y_{V}\right|\right)$ in (2.6) (and $\exp \left(\beta(\mu-1)\left|Y_{V}^{(l)}\right|\right)$ in (2.12)) may be omitted, and we use the previous notation for the partition functions with this factor canceled.

Denoting, for a classical contour $\gamma=(\widetilde{\gamma}, E, I)$,

$$
\|\gamma\|=|\widetilde{\gamma}| \cdot|E-I|
$$

and for a compatible collection of contours $\left\{\gamma_{j}\right\}$,

$$
\left\|\left\{\gamma_{j}\right\}\right\|=\sum_{j}\left\|\gamma_{j}\right\|
$$

we obtain, by virtue of (2.8), the following lemma.

Lemma 2.2. The statistical weight of a quantum contour $\zeta$ equals

$$
w(\boldsymbol{\zeta})=\exp \left(-\beta(\mu-1) \frac{1}{2 d}\left\|\left\{\boldsymbol{\gamma}_{j}^{0}(\boldsymbol{\zeta})\right\}\right\|\right)\left(\frac{\beta}{2 d}\right)^{n(\boldsymbol{\zeta})} \frac{1}{n(\boldsymbol{\zeta}) !} \prod_{i=0}^{n(\boldsymbol{\zeta})-1} X^{i}\left(y_{i}^{-}\right)
$$

where $X^{i}=X^{i}(\mathbf{X}(\zeta))$. The partition function $\Xi^{(l)}(V)$, for any finite $V$ and $l=$ $0, \ldots, m$, equals

$$
\Xi^{(l)}(V)=\sum_{\substack{\left\{\boldsymbol{\zeta}_{r}\right\}:\left\{\tilde{\zeta}_{r}\right\} \subset V, X_{V}^{s}\left(\mathbf{X}\left(\left\{\boldsymbol{\zeta}_{r}\right\}\right)\right)+Y_{V^{c}}^{(l)} \\ \text { is admissible }}} \prod_{r} w\left(\boldsymbol{\zeta}_{r}\right) .
$$


Formula (2.16) provides the so-called contour representation of the partition function: it allows us to introduce the ensemble of quantum contours in $V$. This is the starting point of a polymer expansion used for proving Theorems 1 and 2 . In the next section we derive an appropriate scheme leading to this expansion, and in Section 4 complete the proof.

\section{A polymer expansion for abstract contour models and its application to contour models on $\mathbb{Z}^{d}$}

Formally, the content of this section does not depend on that of Sections 1 and 2. However, we use a notation and terminology that provide a transparent connection with the preceding section and serve as a prototype for Section 4.

Abstract contours. Consider a countable set $\Theta$ the elements of which are called (abstract) contours and denoted $\theta, \theta^{\prime}$, ets. A finite subset of $\Theta$ is denoted by $\Lambda$. We fix some reflexive and symmetric relation $\chi$ on $\Theta \times \Theta$. A pair $\theta, \theta^{\prime} \in \Theta$ is called incompatible if $\theta \nsim \theta^{\prime}$ and compatible if $\theta$ not $\psi \theta^{\prime}$ (i.e. $\theta \sim \theta^{\prime}$ ). A collection $\left\{\tilde{\gamma}_{i}\right\}$ of contours is called compatible if any pair of its contours is compatible. We assign to every contour $\theta$ a (generally speaking) complex-valued statistical weight $w(\theta)$, and for every $\Lambda$ define an (abstract) partition function

$$
Z(\Lambda)=\sum_{\left\{\theta_{i}\right\} \subseteq \Lambda} \prod_{i} w\left(\theta_{i}\right)
$$

where the sum is extended to all compatible collections of contours $\theta_{i} \in \Lambda$. The empty collection is compatible by definition, and it is included in $Z(\Lambda)$ with statistical weight 1. A polymer $\pi$ is an (unordered) finite collection, $\pi=\left[\widetilde{\gamma}_{i}^{\alpha_{i}}\right]$, of different contours $\theta_{i} \in \Theta$ taken with positive integer multiplicities $\alpha_{i}$, such that for every pair $\theta^{\prime}, \theta^{\prime \prime} \in \pi$ there exists a sequence $\theta^{\prime}=\theta_{i_{1}}, \theta_{i_{2}}, \ldots, \theta_{i_{k}}=\theta^{\prime \prime} \in \pi$ with $\theta_{i_{j}} \nsim \theta_{i_{j+1}}, 1 \leq j<k$. The notation $\pi \subseteq \Lambda$ used below is self-explanatory. Our result can be formulated as follows.

Theorem 3.1. Suppose that there exists a function $a: \Theta \mapsto \mathbb{R}^{+}$such that statistical weight $w$ obeys, for each contour $\theta$,

$$
\sum_{\theta^{\prime}: \theta^{\prime} \nsim \theta}\left|w\left(\theta^{\prime}\right)\right| \exp \left(a\left(\theta^{\prime}\right)\right) \leq a(\theta)
$$

Then, for any finite $\Lambda$,

$$
\log Z(\Lambda)=\sum_{\pi \subseteq \Lambda} r(\pi) w(\pi)
$$

where

(i) the statistical weight of a polymer $\pi=\left[\widetilde{\gamma}_{i}^{\alpha_{i}}\right]$ equals

$$
w(\pi)=\prod_{i} w\left(\theta_{i}\right)^{\alpha_{i}}
$$


(ii) the combinatorial factor $r(\pi)$ is calculated as

$$
r(\pi)=\prod_{i}\left(\alpha_{i} !\right)^{-1} \sum_{G(\pi)}(-1)^{|G(\pi)|}
$$

Here the sum $\sum_{G(\pi)}$ is taken over all connected graphs $G(\pi)$, with $\sum_{i} \alpha_{i}$ vertices $v_{i, j}(G(\pi)), \quad 1 \leq j \leq \alpha_{i}$, which are labeled by the contours $\theta_{i}$ from $\pi$ counted with their multiplicities, such that an edge can link two vertices, $v(G(\pi))$ and $v^{\prime}(G(\pi))$, only if the corresponding contours are incompatible, and $|G(\pi)|$ denotes the number of the edges in $G(\pi)$.

Moreover, the series (3.3) for $\log Z(\Lambda)$ is absolutely convergent, and for any contour $\theta$

$$
\sum_{\pi: \pi \ni \theta}|r(\pi) w(\pi)| \leq|w(\theta)| \exp (a(\theta)) .
$$

Analogously, for any polymer $\pi=\left[\widetilde{\gamma}_{i}^{\alpha_{i}}\right]$,

$$
\sum_{\pi^{\prime}: \pi^{\prime} \supseteq \pi}\left|r\left(\pi^{\prime}\right) w\left(\pi^{\prime}\right)\right| \leq|w(\pi)| \exp \left(\sum_{i} \alpha_{i} a\left(\theta_{i}\right)\right) \sum_{T(\pi)} 1,
$$

where graph $T(\pi)$ is defined like $G(\pi)$, with an additional requirement to be a tree.

Remark. The meaning of Theorem 3.1 which distinguishes it from other results on general polymer expansions (see, e.g., [ M1 ], [ $\mathrm{KoPr}],[\mathrm{S}],[\mathrm{MMi}]$ ) is that it combines a convenient condition (3.2) of the absolute convergence of the expansion (3.3) with a standard form of the polymers and their statistical weights. [In the references above, one or another of these tasks was not fully accomplished. For example, in [ $\mathrm{KoPr}]$ (where condition (3.2) was introduced in the first instance), the polymer expansion is established in terms of partition functions in subsets $\Lambda^{\prime} \subseteq \Lambda$; this is not convenient when one is interested in the detailed properties of the model.]

Proof. Formula (3.3) for $\log Z(\Lambda)$ can be easily obtained as a result of calculations with the formal series $[S]$, and the only question is the convergence of the series in the right-hand side of (3.3). It can be verified that for every $\pi=\left[\widetilde{\gamma}_{i}^{\alpha_{i}}\right]$

$$
\left|\sum_{G(\pi)}(-1)^{|G(\pi)|}\right| \leq \sum_{T(\pi)} 1
$$

(see $[\mathrm{S}]$ ). Owing to (3.8), we have the following bound

$$
\sum_{\pi: \pi \ni \theta}|r(\pi) w(\pi)| \leq \sum_{\pi: \pi \ni \theta} \sum_{T(\pi)} \prod_{i}\left(\alpha_{i} !\right)^{-1}\left|w\left(\theta_{i}\right)\right|^{\alpha_{i}}
$$

Observe that quantity in the right-hand side of (3.9) is equal to

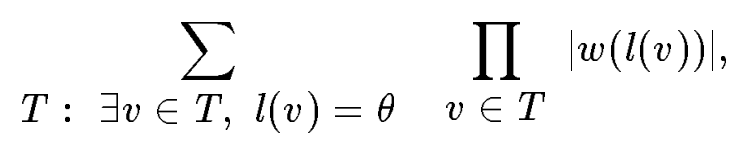


where the sum is over finite labeled trees $T$ with the following properties:

(i) for every vertex $v \in T$, there is a contour $\theta^{\prime}$ associated with $v$ (we write this as $\theta^{\prime}=l(v)$ and call $\theta^{\prime}$ a label of vertex $\left.v\right)$;

(ii) for any edge of $T$ the contours labeling the vertices adjacent to this edge are incompatible;

(iii) there exists a vertex $v \in T$ labeled by $\theta$.

Note that two labeled trees are considered isomorphic (and not distinguished) iff there is a 1-1 map, from the vertex set of one labeled tree onto that of another one which preserves both the adjacency and the labeling.

For every labeled tree $T$ we fix one of the vertices, with label $\theta$ as a root $r(T)$. Such trees are called $\theta$-rooted labeled trees. For a given $\theta$-rooted labeled tree $T$ denote by $\alpha(T)$ the total number of vertices of $T$ labeled by $\theta$. Expression (3.10) is then equal to

$$
\sum_{T: l(r(T))=\theta} \frac{1}{\alpha(T)} \prod_{v \in T}|w(l(v))|,
$$

where the sum is over the $\theta$-rooted labeled trees. The factor $\frac{1}{\alpha(T)}$ appears because two $\theta$-rooted labeled trees are now considered the same iff there is a 1-1 map from the the vertex set of one tree onto that of another one which takes the root of one tree to that of another one and preserves both the adjacency and the labeling. Obviously, (3.11) is not greater than

$$
\begin{gathered}
|w(\theta)| \sum_{T: l(r(T))=\theta} \prod_{v \in T, v \neq r(T)}|w(l(v))| \\
=|w(\theta)| \sum_{T: l(r(T))=\theta} w(T)
\end{gathered}
$$

where

$$
w(T)=\prod_{v \in T, v \neq r(T)}|w(l(v))| .
$$

We define the level of a vertex $v$ of a rooted tree $T$ as the length of the path from $r(T)$ to $v$; the highest level $n(T)$ in $T$ is the maximal length of the paths from $r(T)$ to the leaves of $T$. In the sequel, when speaking about an edge $\left(v, v^{\prime}\right)$ of a rooted tree $T$, we assume that the level of vertex $v$ (the beginning of the edge) is one less than the level of vertex $v^{\prime}$ (the end of the edge).

The edges of $\theta$-rooted tree $T$ can be uniquely decomposed into bunches. A bunch is defined as a set of edges having a common beginning, at some vertex $v$, and a single contour $\theta^{\prime}$ labeling their ends (we say that a bunch grows from $v$ and is labeled by $\theta^{\prime}$ ). To make the partition unique, one should include, in every bunch, a maximal possible number of edges. We denote a bunch by $b=\left\langle v, \theta^{\prime}, \alpha^{\prime}\right\rangle$, where $\alpha^{\prime}=\alpha^{\prime}(b)$ is the number of edges in the bunch. We also use a short-hand notation $b=\left\langle\theta^{\prime}, \alpha^{\prime}\right\rangle$ when it is clear or not important what initial vertex $v$ is meant. Our definition of the bunch implies that, for given contour $\theta^{\prime}$ and vertex $v$, not more than one bunch labeled by $\theta^{\prime}$ can grow from $v$. 
Every $\theta$-rooted labeled tree can be obtained as a result of the following procedure of growing bunches. To form the level-one part of a $\theta$-rooted labeled tree, we fix a finite collection of contours $\left\{\theta_{i}^{\prime}\right\}, \theta_{i}^{\prime} \nsim \theta$, and corresponding values $\alpha_{i}^{\prime} \geq 1$ and join the set of bunches $b_{i}=\left\langle r, \theta_{i}^{\prime}, \alpha_{i}^{\prime}\right\rangle, i=1,2, \ldots$, with the root $r$ labeled by $\theta$. To form the level-two part, we repeat this procedure with the ends of every bunch constructed at the previous step. That is, for a given end $v$ labeled by $\theta_{i}^{\prime}$, we choose a finite collection of contours $\left\{\theta_{j}^{\prime \prime}\right\}, \theta_{j}^{\prime \prime} \nsucc \theta_{i}^{\prime}$, and corresponding values $\alpha_{j}^{\prime \prime} \geq 1$ and join the set of bunches $b_{j}=\left\langle v, \theta_{j}^{\prime \prime}, \alpha_{j}^{\prime \prime}\right\rangle, j=1,2, \ldots$, with vertex $v$. Repeating the procedure several times, we can construct any finite $\theta$-rooted labeled tree. Observe that for any $\theta$-rooted labeled tree $T$, with a collection of bunches $b_{k}(T)=\left\langle\alpha_{k}(T), \theta_{k}(T)\right\rangle, k=1,2, \ldots$, our procedure produces $\prod_{k} \alpha_{k}(T)$ ! isomorphic copies of $T$ because it treats the vertices of given bunch as distinct. In fact, any permutation of the edges of a bunch gives us an isomorphic copy of the $\theta$-rooted labeled tree.

We take the statistical weight of bunch $b=\left\langle\theta^{\prime}, \alpha^{\prime}\right\rangle$ to be equal

$$
w(b)=\frac{\left|w\left(\theta^{\prime}(b)\right)\right|^{\alpha^{\prime}(b)}}{\alpha^{\prime}(b) !}
$$

and denote

$$
f(\theta, k)=\sum_{T: l(r(T))=\theta, n(T) \leq k} w(T),
$$

where $n(T)$ is the number of levels in $T$. By induction in $m$ we prove below that

$$
f(\theta, k) \leq \exp (a(\theta)) \text {. }
$$

First we estimate the quantity $f(\theta, 1)$. According to the initial step of the bunch-growing procedure,

$$
\begin{aligned}
f(\theta, 1) & \sum_{T: l(r(T))=\theta, n(T) \leq 1} w(T) \\
& =\prod_{\theta^{\prime} \nsim \theta} \sum_{\alpha^{\prime}=0}^{\infty} w\left(\left\langle\theta^{\prime}, \alpha^{\prime}\right\rangle\right) \\
& =\prod_{\theta^{\prime} \nsim \theta} \exp \left(\left|w\left(\theta^{\prime}\right)\right|\right) \\
& =\exp \left(\sum_{\theta^{\prime} \nsim \theta}\left|w\left(\theta^{\prime}\right)\right|\right) .
\end{aligned}
$$

By virtue of (3.2), $\sum_{\theta^{\prime} \nsim \theta}\left|w\left(\theta^{\prime}\right)\right| \leq a(\theta)$, and hence

$$
f(\theta, 1) \leq \exp (\alpha(\theta)) .
$$

Assume now that

$$
f(\theta, k-1) \leq \exp (a(\theta)) .
$$


Again using the bunch-growing procedure, one can write a recursive representation for the right-hand side of (3.15):

$$
f(\theta, k)=\prod_{\theta^{\prime} \not \theta} \sum_{\alpha^{\prime}=0}^{\infty} w\left(\left\langle\theta^{\prime}, \alpha^{\prime}\right\rangle\right) f\left(\theta^{\prime}, k-1\right)^{\alpha^{\prime}} .
$$

Using the induction hypothesis for $f(\theta, k-1)$, we obtain

$$
\begin{aligned}
f(\theta, k) & =\prod_{\theta^{\prime} \neq \theta} \sum_{\alpha^{\prime}=0}^{\infty} w\left(\left\langle\theta^{\prime}, \alpha^{\prime}\right\rangle\right) f\left(\theta^{\prime}, k-1\right)^{\alpha^{\prime}} \\
& \leq \prod_{\theta^{\prime} \nsim \theta} \sum_{\alpha^{\prime}=0}^{\infty} w\left(\left\langle\theta^{\prime}, \alpha^{\prime}\right\rangle\right) \exp \left(\alpha^{\prime} a\left(\theta^{\prime}\right)\right) \\
& =\prod_{\theta^{\prime} \nsim \theta} \exp \left(\left|w\left(\theta^{\prime}\right)\right| \exp \left(a\left(\theta^{\prime}\right)\right)\right) \\
& =\exp \left(\sum_{\theta^{\prime} \nsim \theta}\left|w\left(\theta^{\prime}\right)\right| \exp \left(a\left(\theta^{\prime}\right)\right)\right) \\
& \leq \exp (a(\theta)),
\end{aligned}
$$

which proves (3.6). Bound (3.7) can be proved in an analogous way.

We will apply general Theorem 3.1 to various contour models on lattice $\mathbb{Z}^{d}$. Our aim here is to derive some general assertions, which are then applied to the specific situation of the quantum system under consideration. The corresponding contour model deals with complicated geometrical objects, and to make the whole exposition easier, we begin with somewhat simpler models. More precisely, we derive a series of statements for contour models of increasing complexity, with each model being a natural generalization of the previous one.

Contour model 1. We start with the following model. Let bulky contour $\widetilde{\Omega}$ be defined as a finite connected subset of lattice $\mathbb{Z}^{d}, d \geq 2$. Define the boundary $\partial \widetilde{\Omega}$ as the set of unit plaquettes of dual lattice $\tilde{\mathbb{Z}}^{d}$ separating $\widetilde{\Omega}$ and its complement $\widetilde{\Omega}^{c}=\mathbb{Z}^{d} \backslash \widetilde{\Omega}$. Then $\partial \widetilde{\Omega}$ can be uniquely decomposed in connected components $\partial_{i} \widetilde{\Omega}, i=$ $0,1, \ldots, n(\widetilde{\Omega})$, alternatively denoted by $\widetilde{\omega}_{i}(\tilde{\Omega})$. We always assume that $\widetilde{\omega}_{0}(\widetilde{\Omega})$ stands for the (unique) external component of $\partial \widetilde{\Omega}$.

We say that $\widetilde{\Omega}$ and $\widetilde{\Omega}^{\prime}$ are compatible if $\widetilde{\Omega} \cap \widetilde{\Omega}^{\prime}=\emptyset$. A finite collection $\left\{\widetilde{\Omega}_{i}\right\}$ is called compatible if any pair $\widetilde{\Omega}_{i_{1}}, \widetilde{\Omega}_{i_{2}}$ with $i_{1} \neq i_{2}$ is compatible.

Assign to $\widetilde{\Omega}$ a statistical weight $w(\widetilde{\Omega})$ and suppose that it obeys

$$
|w(\tilde{\Omega})| \leq \exp \left(-p \sum_{i=0}^{n(\tilde{\Omega})}\left|\widetilde{\omega}_{i}(\tilde{\Omega})\right|-q|\widetilde{\Omega}|\right)
$$

where $p, q>0$ are constants. Here and below $\left|\widetilde{\omega}_{i}\right|$ is the number of plaquettes in $\widetilde{\omega}_{i}$ and $|\widetilde{\Omega}|$ the number of lattice sites in $\widetilde{\Omega}$. 
For a finite $V \subset \mathbb{Z}^{d}$, define the partition function in $V$ by

$$
Z(V)=\sum_{\left\{\widetilde{\Omega}_{j}\right\} \subseteq V} \prod_{j} w\left(\left\{\tilde{\Omega}_{j}\right\}\right)
$$

Here, the sum $\sum_{\tilde{\Omega}_{j} \subseteq V}$ is over the compatible collections $\left\{\tilde{\Omega}_{j}\right\}$, where each $\tilde{\Omega}_{i} \subseteq V$.

Contour models of such type usually serve to describe two-phase physical models, with one phase dominating another. The simplest example is the Ising model with the external magnetic field. To see the correspondence, suppose that the field is positive and fix the + boundary condition. Then bulky contours $\widetilde{\Omega}$ are connected clusters of the -'s in a sea of the +'s. Parameters $p, q$ in this case are the coupling constant and the magnetic field, respectively.

The following statement is a corollary of Theorem 3.1 .

Proposition 3.2. Under condition (3.22), with

$$
p \geq 2+\log (2 d), q \geq 8 d \exp (-p / 2),
$$

the logarithm of partition function (3.23) admits a polymer expansion of form (3.3), where properties (3.6), (3.7) are valid, with

$$
a(\widetilde{\Omega})=h|\widetilde{\Omega}|, h=2 d \exp (-p / 2) .
$$

Proof. According to Theorem 3.1, it is sufficient to verify that $a(\tilde{\Omega})$ given by (3.24) satisfies condition (3.2).

To carry out the summation in $(3.2)$, we provide every $\widetilde{\Omega}$ with a tree-like structure. For this purpose we consider $\widetilde{\Omega}$ as a closed domain in $\mathbb{R}^{d}$, i.e. as the union of the unit cubes of $\mathbb{Z}^{d}$ centered at the points of $\widetilde{\Omega}$. Next we mark, in each connected component $\widetilde{\omega}_{i}$ of boundary $\partial \widetilde{\Omega}$, the center of an arbitrarily selected plaquette and through each marked point draw a line parallel to the first coordinate axis $L$. Together with $\partial \widetilde{\Omega}$, the union of these lines represents a connected subset of $\mathbb{R}^{d}$. Every line is partitioned by $\partial \widetilde{\Omega}$ into intervals. As the final step of the construction, we choose, in any possible way, a minimal number of the intervals such that, being united with $\partial \widetilde{\Omega}$, these intervals still form a connected subset of $\mathbb{R}^{d}$. Obviously, all selected intervals belong to $\widetilde{\Omega}$ treated as a subset of $\mathbb{R}^{d}$.

The object just constructed has a tree-like structure in the following sense. The component $\widetilde{\omega}_{0}(\widetilde{\Omega})$ is by definition the root of the tree. The components $\widetilde{\omega}_{1, k}(\widetilde{\Omega})$ which are linked, by means of selected intervals, directly with $\widetilde{\omega}_{0}(\widetilde{\Omega})$, are the vertices of the first level (from now on we use the double-indexed numeration for the components of $\partial \widetilde{\Omega})$. The components $\tilde{\omega}_{j, k}(\widetilde{\Omega})$ which are linked by means of intervals directly with $\cup_{k} \widetilde{\omega}_{j-1, k}(\tilde{\Omega})$ are the vertices of the $j$-th level. Denote by $L_{j, k}$ the interval linking $\widetilde{\omega}_{j, k}(\widetilde{\Omega})$ with $\cup_{k} \widetilde{\omega}_{j-1, k}(\widetilde{\Omega})$ and denote by $\left|L_{j, k}\right|$ the number of points of $\mathbb{Z}^{d}$ belonging to $L_{j, k}$. We treat the intervals as the edges of our tree. The bound

$$
|w(\widetilde{\Omega})| \leq \exp \left(-(p+q /(4 d))\left|\tilde{\omega}_{0}(\tilde{\Omega})\right|-(p+q /(4 d)) \sum_{j, k}\left|\widetilde{\omega}_{j, k}(\tilde{\Omega})\right|-q / 2 \sum_{j, k}\left|L_{j, k}\right|\right)
$$


follows from (3.22), because, by construction,

$$
\sum_{j, k}\left|L_{j, k}\right| \leq|\widetilde{\Omega}| \text { and } 2 d|\widetilde{\Omega}| \geq \sum_{j, k}\left|\widetilde{\omega}_{j, k}(\widetilde{\Omega})\right|+\left|\widetilde{\omega}_{0}(\widetilde{\Omega})\right|
$$

Now consider $\widetilde{\Omega}$ as a tree-like object, $T=\left(\widetilde{\omega}_{0},\left\{\widetilde{\omega}_{j, k}, L_{j, k}\right\}\right)$, where $\widetilde{\omega}_{0}$ and $\widetilde{\omega}_{j, k}$ are finite connected sets of plaquettes of $\widetilde{\mathbb{Z}}^{d}$ and $L_{j, k}$ are the intervals parallel to $L$ (see above); $L_{j, k}$ links the center of a plaquette from $\widetilde{\omega}_{j, k}$ with the center of a plaquette from $\cup_{k} \widetilde{\omega}_{j-1, k}$. Assign to every $T$ the statistical weight

$$
w(T)=\exp \left(-(p+q /(4 d))\left|\widetilde{\omega}_{0}\right|-(p+q /(4 d)) \sum_{j, k}\left|\widetilde{\omega}_{j, k}\right|-q / 2 \sum_{j, k}\left|L_{j, k}\right|\right)
$$

and set

$$
a(T)=h \sum_{j, k}\left|L_{j, k}(T)\right|
$$

Then, for every fixed $\widetilde{\omega}$,

$$
\sum_{\widetilde{\Omega}: \widetilde{\omega}_{0}(\widetilde{\Omega})=\widetilde{\omega}}|w(\widetilde{\Omega})| \exp (a(\tilde{\Omega})) \leq \sum_{T: \widetilde{\omega}_{0}(T)=\widetilde{\omega}} w(T) \exp (a(T))=S(\widetilde{\omega}),
$$

because $h \leq \frac{q}{4}$.

Lemma 3.3 The following bound holds true:

$$
\sum_{T: \widetilde{\omega}_{0}(T)=\widetilde{\omega}} w(T) \exp (a(T)) \leq \exp (-p|\widetilde{\omega}|)
$$

Proof of Lemma 3.3. We proceed by induction on the number of levels $n(T)$ in $T$. Suppose that, for some $J \geq 1$, the bound

$$
T: \tilde{\omega}_{0}(T)=\widetilde{\omega}, n(T) \leq J-1
$$

has been established, and consider an arbitrary $T$ with $n(T) \leq J$. For a given vertex $\tilde{\omega}_{1, k}$ of the first level in $T$, denote by $T_{1, k}$ the maximal subtree of $T$ that has its root at $\tilde{\omega}_{1, k}$. Then

$$
\begin{aligned}
S(\widetilde{\omega})= & \sum w(T) \exp (a(T)) \\
= & \exp [-(p+q /(4 d))|\widetilde{\omega}|] \sum_{s=0}^{|\widetilde{\omega}|}\left(\begin{array}{c}
|\widetilde{\omega}| \\
s
\end{array}\right) \\
& \times \prod_{k=1}^{s}\left(\sum_{\left|L_{1, k}\right|=1}^{\infty} \exp \left(-(q / 2-h)\left|L_{1, k}\right|\right)\right. \\
& \times \sum_{\widetilde{\omega}_{1, k} T_{1, k}: \tilde{\omega}_{0}\left(T_{1, k}\right)=\widetilde{\omega}_{1, k},}^{n\left(T_{1, k}\right) \leq J-1},
\end{aligned}
$$


where $s$ is the number of the vertices of the first level in $T$ and $\left(\begin{array}{c}|\widetilde{\omega}| \\ s\end{array}\right)$ describes the ways to choose the centers of plaquettes which serve as the starting points of intervals $L_{1, k}, k=1, \ldots, s$. Applying the induction assumption to the third sum in (3.31), we obtain

$$
\begin{aligned}
& S(\widetilde{\omega}) \leq \exp [-(p+q /(4 d))|\widetilde{\omega}|] \sum_{s=0}^{|\widetilde{\omega}|}\left(\begin{array}{c}
|\widetilde{\omega}| \\
s
\end{array}\right) \\
& \times \prod_{k=1}^{s}\left(\sum_{\left|L_{1, k}\right|=1}^{\infty} \exp \left[-(q / 2-h)\left|L_{1, k}\right|\right] \sum_{\widetilde{\omega}_{1, k}} \exp \left(-p\left|\widetilde{\omega}_{1, k}\right|\right)\right) \\
& \leq \exp [-(p+q /(4 d))|\widetilde{\omega}|] \sum_{s=0}^{|\widetilde{\omega}|}\left(\begin{array}{c}
|\widetilde{\omega}| \\
s
\end{array}\right) \\
& \times \prod_{k=1}^{s}\left(\frac{2 d \exp (-p)}{1-2 d \exp (-p)} \sum_{\left|L_{1, k}\right|=1}^{\infty} \exp \left[-(q / 2-h)\left|L_{1, k}\right|\right]\right) \\
& \leq \exp [-(p+q /(4 d))|\widetilde{\omega}|] \sum_{s=0}^{|\widetilde{\omega}|}\left(\begin{array}{c}
|\widetilde{\omega}| \\
s
\end{array}\right) \\
& \times \prod_{k=1}^{s}\left(\frac{2 d \exp (-p)}{1-2 d \exp (-p)} \cdot \frac{\exp (-(q / 2-h))}{1-\exp (-(q / 2-h))}\right) \\
& \leq \exp [-(p+q /(4 d))|\widetilde{\omega}|] \sum_{s=0}^{|\widetilde{\omega}|}\left(\begin{array}{c}
|\widetilde{\omega}| \\
s
\end{array}\right) \\
& \times\left(\frac{2 d \exp (-p)}{1-2 d \exp (-p)} \cdot \frac{\exp (-(q / 2-h))}{1-\exp (-(q / 2-h))}\right)^{s} \\
& \leq \exp [-(p+q /(4 d))|\widetilde{\omega}|] \\
& \times\left(1+\frac{2 d \exp (-p)}{1-2 d \exp (-p)} \cdot \frac{\exp (-(q / 2-h))}{1-\exp (-(q / 2-h))}\right)^{|\widetilde{\omega}|} \\
& \leq \exp (-p|\widetilde{\omega}|) \text {. }
\end{aligned}
$$

The last bound is valid because for $p \geq 2+\log (2 d)$ and $h \leq \frac{q}{4}$ we have

$$
1-2 d \exp (-p) \geq \frac{1}{2}
$$

and hence

$$
\begin{aligned}
1+\frac{2 d \exp (-p)}{1-2 d \exp (-p)} \cdot \frac{\exp (-(q / 2-a))}{1-\exp (-(q / 2-a))} & \leq 1+\frac{4 d \exp (-p)}{q / 2-a} \\
& \leq 1+\frac{16 d \exp (-p)}{q} \\
& \leq 1+q /(4 d) \\
& \leq \exp (q /(4 d))
\end{aligned}
$$


To establish the initial step of the induction, observe that, for $T$ with $n(T)=0$, we obviously have $w(T)=\exp (-p|\widetilde{\omega}|)$.

Now we can check condition (3.2):

$$
\begin{aligned}
& \sum_{\tilde{\Omega}: \tilde{\Omega}^{\prime} \neq \widetilde{\Omega}}\left|w\left(\tilde{\Omega}^{\prime}\right)\right| \exp \left(a\left(\tilde{\Omega}^{\prime}\right)\right)=\sum_{\tilde{\omega}^{\prime}: \tilde{\omega}^{\prime} \cap \tilde{\Omega} \neq \emptyset \tilde{\Omega}^{\prime}: w_{0}\left(\tilde{\Omega}^{\prime}\right)=\tilde{\omega}^{\prime}}\left|w\left(\tilde{\Omega}^{\prime}\right)\right| \exp \left(a\left(\tilde{\Omega}^{\prime}\right)\right) \\
& \leq \sum_{\widetilde{\omega}^{\prime}: \tilde{\omega}^{\prime} \cap \tilde{\Omega} \neq \emptyset} \exp \left(-p\left|\widetilde{\omega}^{\prime}\right|\right) \\
& \leq|\widetilde{\Omega}| \frac{2 d \exp (-p)}{1-2 d \exp (-p)} \\
& \leq 4 d \exp (-p)|\widetilde{\Omega}| \\
& \leq 2 d \exp (-p / 2)|\widetilde{\Omega}| \\
& =h|\widetilde{\Omega}| \text {. }
\end{aligned}
$$

This completes the proof of Proposition 3.2.

Contour model 2. The idea behind our second model is that the difference in the statistical weight of lowest exitations can lead to an asymmetry between two phases, in the same way as the external field does. [An example of a model of this kind is the so-called ANNNI model (see, e.g., [ DMaSin ])]. We now define a bulky contour $\widetilde{\Omega}$ as a finite connected subset of $\mathbb{Z}^{d}$, with the additional condition

$$
\operatorname{diam}\left|\widetilde{\omega}_{i}(\widetilde{\Omega})\right| \geq u>1
$$

and consider a statistical weight $W(\widetilde{\Omega})$ satisfying

$$
|W(\widetilde{\Omega})| \leq \exp \left(-p \sum_{i=0}^{n(\tilde{\Omega})}\left|\widetilde{\omega}_{i}(\tilde{\Omega})\right|\right)
$$

Moreover, we suppose that, in addition, the model contains other objects denoted $\widetilde{\varepsilon}^{+}$and $\widetilde{\varepsilon}^{-}$and called short contours. Both $\widetilde{\varepsilon}^{+}$and $\widetilde{\varepsilon}^{-}$are finite connected subset of dual lattice $\tilde{\mathbb{Z}}^{d}$ such that they are the boundaries of connected and simpleconnected subsets of lattice $\mathbb{Z}^{d}$, with

$$
\operatorname{diam} \widetilde{\varepsilon}^{ \pm}<u
$$

The difference between $\widetilde{\varepsilon}^{+}$and $\widetilde{\varepsilon}^{-}$is twofold. First, it is manifested in the modified compatibility rule for the possible pairs $\left(\widetilde{\Omega}, \widetilde{\Omega}^{\prime}\right),\left(\widetilde{\Omega}, \widetilde{\varepsilon}^{ \pm}\right)$and $\left(\widetilde{\varepsilon}^{ \pm}, \widetilde{\varepsilon}^{\prime \pm}\right)$, which now requires

(i) $\widetilde{\Omega} \cap \widetilde{\Omega}^{\prime}=\emptyset$;

(ii.1) $\widetilde{\varepsilon}^{ \pm} \cap \widetilde{\omega}_{i}(\widetilde{\Omega})=\emptyset$,

(ii.2) $\widetilde{\varepsilon}^{-} \cap \widetilde{\Omega}^{c}=\emptyset$,

(ii.3) $\widetilde{\varepsilon}^{+} \cap \widetilde{\Omega}=\emptyset$; 
(iii) $\widetilde{\varepsilon}^{\prime \pm} \cap \widetilde{\varepsilon}^{\prime \prime \pm}=\emptyset$;

The definition of a compatible collection $\left\{\widetilde{\Omega}_{i}, \widetilde{\varepsilon}_{j}^{+}, \widetilde{\varepsilon}_{k}^{-}\right\}$is based, as before, on pairwise compatibility.

Secondly, the statistical weights of $\widetilde{\varepsilon}^{ \pm}$are small:

$$
\left|w\left(\widetilde{\varepsilon}^{ \pm}\right)\right| \leq \exp \left(-p\left|\widetilde{\varepsilon}^{ \pm}\right|\right),
$$

and are different for $\widetilde{\varepsilon}^{+}$and $\widetilde{\varepsilon}^{-}$. More precisely, we assume that for any finite $V$,

$$
\frac{Z^{-}(V)}{Z^{+}(V)} \leq \exp [-|V| 4 d \exp (-(p-2)(d u-u+1))],
$$

where the restricted (or metastable) partition functions $Z^{ \pm}(V)$ are given by

$$
Z^{ \pm}(V)=\sum_{\left\{\widetilde{\varepsilon}_{i}^{ \pm}\right\} \subseteq V} \prod_{i} w\left(\widetilde{\varepsilon}_{i}^{ \pm}\right)
$$

Here the sum $\sum_{\left\{\widetilde{\varepsilon}_{i}^{ \pm}\right\} \subseteq V}$ is over the compatible collections of the $\widetilde{\varepsilon}^{+}$'s or $\widetilde{\varepsilon}$ 's (all of the same sign), where each $\widetilde{\varepsilon}_{j}$ is contained in set $V$ treated as a closed domain in $\mathbb{R}^{d}$ (in the same way as before (see p.17)). We will briefly say that $\widetilde{\varepsilon}_{j}$ is contained in $V$ in the $\mathbb{R}^{d}$-sense. Although condition (3.40) looks dificult to check, it may be verified in many situations where bound (3.39) is valid. One method of proving the validity of $(3.40)$ is to use Theorem 3.1 (more precisely formula (3.3) for $\log Z^{ \pm}(V)$ ), with a direct bound for the difference of statistical weights of corresponding polymers.

The full partition function of the model is defined as

$$
Z(V)=\sum_{\left\{\widetilde{\Omega}_{i}, \widetilde{\varepsilon}_{j}^{+}, \widetilde{\varepsilon}_{k}\right\} \subseteq V} \prod_{i} W\left(\widetilde{\Omega}_{i}\right) \prod_{j} w\left(\widetilde{\varepsilon}_{j}^{+}\right) \prod_{k} w\left(\widetilde{\varepsilon}_{k}\right) .
$$

The sum $\quad \sum \quad$ is over the compatible collections of the $\tilde{\Omega}^{\prime}$ s, $\widetilde{\varepsilon}^{+}$'s and $\left\{\widetilde{\Omega}_{i}, \widetilde{\varepsilon}_{j}^{+}, \widetilde{\varepsilon_{k}}\right\} \subseteq V$

$\widetilde{\varepsilon}$ 's, where each $\widetilde{\Omega}_{i} \subseteq V$ in a usual sense and each $\widetilde{\varepsilon}_{j}^{+}$and $\widetilde{\varepsilon_{k}}$ is contained in $V$ in the $\mathbb{R}^{d}$-sense. [The same meaning is used below, unless otherwise specified.]

Proposition 3.4 Under conditions (3.37) - (3.40), with

$$
p \geq 4+\log (2 d)
$$

the logarithm of partition function (3.42) admits a polymer expansion of form (3.3), where properties (3.6), (3.7) are valid with

$$
a(\widetilde{\varepsilon})=|\widetilde{\varepsilon}|, \quad a(\widetilde{\Omega})=\sum_{i=0}^{n(\widetilde{\Omega})}\left|\widetilde{\omega}_{i}(\widetilde{\Omega})\right|+h|\widetilde{\Omega}| .
$$

and $h=d \exp (-(p-2)(d u-u+1))$. 
Proof. We will show that the current lattice contour model is similar to model 1. Indeed,

$$
\begin{aligned}
Z(V)= & \sum_{\left\{\tilde{\Omega}_{i}\right\} \subseteq V} Z^{+}\left(\left[V \backslash\left(\cup_{i} \tilde{\Omega}_{i}\right)\right]\right) \prod_{i} W\left(\tilde{\Omega}_{i}\right) Z^{-}\left(\left[\tilde{\Omega}_{i}\right]\right) \\
= & \sum_{\left\{\tilde{\Omega}_{i}\right\} \subseteq V} Z^{+}\left(\left[V \backslash\left(\cup_{i} \tilde{\Omega}_{i}\right)\right]\right) \prod_{i} W\left(\tilde{\Omega}_{i}\right) \frac{Z^{-}\left(\left[\tilde{\Omega}_{i}\right]\right)}{Z^{+}\left(\left[\tilde{\Omega}_{i}\right]\right)} Z^{+}\left(\left[\tilde{\Omega}_{i}\right]\right) .
\end{aligned}
$$

Here and below, until otherwise specified, given $D \subset \mathbb{Z}^{d},[D]$ stands for the set $\left\{x \in D: \operatorname{dist}\left(x, D^{c}\right) \geq 1\right\}$. Denoting

$$
w(\tilde{\Omega})=W(\tilde{\Omega}) \frac{Z^{-}([\tilde{\Omega}])}{Z^{+}([\tilde{\Omega}])},
$$

we obtain

$$
\begin{aligned}
Z(V)= & \sum_{\left\{\widetilde{\Omega}_{i}\right\} \subseteq V} Z^{+}\left(\left[V \backslash\left(\cup_{i} \widetilde{\Omega}_{i}\right)\right]\right) \prod_{i} w\left(\widetilde{\Omega}_{i}\right) Z^{+}\left(\left[\widetilde{\Omega}_{i}\right]\right) \\
= & \sum_{\left\{\widetilde{\Omega}_{i}, \widetilde{\varepsilon}_{j}^{+}\right\} \subseteq V} \prod_{i} w\left(\widetilde{\Omega}_{i}\right) \prod_{j} w\left(\widetilde{\varepsilon}_{j}^{+}\right) .
\end{aligned}
$$

In the right-hand side of (3.47) the sum $\sum$ is taken over compatible col$\left\{\widetilde{\Omega}_{i}, \widetilde{\varepsilon}_{j}^{+}\right\} \subseteq V$

lections of bulky contours $\widetilde{\Omega}_{i}$ and short contours $\widetilde{\varepsilon}_{j}^{+}$, with a simplified binary compatibility rule:

(i) $\widetilde{\Omega} \cap \widetilde{\Omega}^{\prime}=\emptyset$;

(ii) $\widetilde{\varepsilon}^{+} \cap \widetilde{\omega}_{i}(\widetilde{\Omega})=\emptyset$;

(iii) $\vec{\varepsilon}^{-1+} \cap \widetilde{\varepsilon}^{-1+}=\emptyset$;

To stress this difference between the compatibility rules, and to simplify the notation, we omit the superscript + . The final form of the partition function is

$$
Z(V)=\sum_{\left\{\widetilde{\Omega}_{i}, \widetilde{\varepsilon}_{j}\right\} \subseteq V} \prod_{i} w\left(\widetilde{\Omega}_{i}\right) \prod_{j} w\left(\widetilde{\varepsilon}_{j}\right)
$$

Now, according to $(3.40)$,

$$
\begin{aligned}
|w(\widetilde{\Omega})| & \leq \exp \left(-p \sum_{i=0}^{n(\tilde{\Omega})}\left|\widetilde{\omega}_{i}(\tilde{\Omega})\right|-q|[\tilde{\Omega}]|\right) \\
& \leq \exp \left(-(p-1) \sum_{i=0}^{n(\tilde{\Omega})}\left|\widetilde{\omega}_{i}(\tilde{\Omega})\right|-q|\widetilde{\Omega}|\right)
\end{aligned}
$$


for $q=4 d \exp (-(p-2)(d u-u+1))<1$. The difference between (3.48) and (3.23) is due only to the presence of short contours, which is easy to deal with. We will check condition (3.2), with $a(\widetilde{\varepsilon})$ and $a(\widetilde{\Omega})$ given by (3.44). Namely, the following bound holds

$$
\begin{aligned}
\sum_{\vec{\varepsilon}: \widetilde{\varepsilon}^{\prime} \neq \widetilde{\varepsilon}} w\left(\widetilde{\varepsilon}^{-1} \exp \left(a\left(\widetilde{\varepsilon}^{\prime}\right)\right)\right. & \leq \sum_{\widetilde{\varepsilon}^{\prime}: \widetilde{\varepsilon}^{-1} \nsucc \widetilde{\varepsilon}} \exp \left(-(p-2)\left|\vec{\varepsilon}^{\prime}\right|\right) \\
& \leq|\widetilde{\varepsilon}| \sum_{n=1}^{\infty}(2 d \exp (-(p-2)))^{n} \\
& \leq \frac{1}{2}|\widetilde{\varepsilon}| .
\end{aligned}
$$

Symbol $\nsim$, here and below, is used to indicate incompatibility in the sense of a current contour model.

On the other hand,

$$
\begin{aligned}
& \sum_{\widetilde{\Omega}^{\prime}: \tilde{\Omega}^{\prime} \nsucc \widetilde{\varepsilon}} w\left(\tilde{\Omega}^{\prime}\right) \exp \left(a\left(\tilde{\Omega}^{\prime}\right)\right) \\
& \leq \sum_{\widetilde{\Omega}^{\prime}: \widetilde{\Omega}^{\prime} \nsim \widetilde{\varepsilon}} \exp \left(-(p-2) \sum_{i=0}^{n\left(\widetilde{\Omega}^{\prime}\right)}\left|\widetilde{\omega}_{i}\left(\tilde{\Omega}^{\prime}\right)\right|-(q-h)\left|\widetilde{\Omega}^{\prime}\right|\right) \\
& \leq \sum_{\tilde{\omega}^{\prime}: \tilde{\omega}^{\prime} \cap \tilde{\varepsilon} \neq \emptyset \tilde{\Omega}^{\prime}: \tilde{\omega}_{0}\left(\widetilde{\Omega}^{\prime}\right)=\widetilde{\omega}^{\prime}} \exp (-(p-2 \\
& \left.+q /(4 d)) \sum_{i=0}^{n\left(\widetilde{\Omega}^{\prime}\right)}\left|\widetilde{\omega}_{i}\left(\tilde{\Omega}^{\prime}\right)\right|-(q / 2-h)\left|\widetilde{\Omega}^{\prime}\right|\right) \\
& \leq \sum_{\widetilde{\omega}^{\prime}: \tilde{\omega}^{\prime} \cap \widetilde{\varepsilon} \neq \emptyset} \exp \left(-(p-2)\left|\widetilde{\omega}^{\prime}\right|\right) \\
& \leq|\widetilde{\varepsilon}| \quad \sum_{n}^{\infty} \quad(2 d \exp (-(p-2)))^{n} \\
& n=2 d u-2 u+2 \\
& \leq \frac{1}{2}|\widetilde{\varepsilon}| \text {. }
\end{aligned}
$$

Here, we used an analogue of Lemma 3.3, with $(p-2)$ instead of $p$. The proof of this statement is similar to that of Lemma 3.3, with the only exception that $(3.32)$ is replaced by

$$
\begin{aligned}
S(\widetilde{\omega}) \leq & \exp [-(p-1+q /(4 d))|\widetilde{\omega}|] \sum_{s=0}^{|\widetilde{\omega}|}\left(\begin{array}{c}
|\widetilde{\omega}| \\
s
\end{array}\right) \\
& \times \prod_{k=1}^{s}\left(\sum_{\left|L_{1, k}\right|=1}^{\infty} \exp \left[-(q / 2-h)\left|L_{1, k}\right|\right] \sum_{\widetilde{\omega}_{1, k}} \exp \left[-(p-2)\left|\widetilde{\omega}_{1, k}\right|\right]\right)
\end{aligned}
$$




$$
\begin{aligned}
& \leq \exp [-(p-1+q /(4 d))|\widetilde{\omega}|] \sum_{s=0}^{|\tilde{\omega}|}\left(\begin{array}{c}
|\widetilde{\omega}| \\
s
\end{array}\right) \\
& \times \prod_{k=1}^{s}\left(\frac{2 d \exp [-(p-2)(2 d u-2 u+2)]}{1-2 d \exp (-p)} \sum_{\left|L_{1, k}\right|=1}^{\infty} \exp \left[-(q / 2-h)\left|L_{1, k}\right|\right]\right) \\
& \leq \exp [-(p-1+q /(4 d))|\widetilde{\omega}|] \sum_{s=0}^{|\widetilde{\omega}|}\left(\begin{array}{c}
|\widetilde{\omega}| \\
s
\end{array}\right) \\
& \times \prod_{k=1}^{s}\left(\frac{2 d \exp [-(p-2)(2 d u-2 u+2)]}{1-2 d \exp (-p)} \cdot \frac{\exp (-(q / 2-h))}{1-\exp (-(q / 2-h))}\right) \\
& \leq \exp [-(p-1+q /(4 d))|\widetilde{\omega}|] \sum_{s=0}^{|\widetilde{\omega}|}\left(\begin{array}{c}
|\widetilde{\omega}| \\
s
\end{array}\right) \\
& \times\left(\frac{2 d \exp [-(p-2)(2 d u-2 u+2)]}{1-2 d \exp (-p)} \cdot \frac{\exp (-(q / 2-h))}{1-\exp (-(q / 2-h))}\right) \\
& \times \exp (-(p-2)|\widetilde{\omega}|) \cdot\left(1+\frac{2 d \exp [-(p-2)(2 d u-2 u+2)]}{1-2 d \exp (-p)} \cdot \frac{\exp (-(q / 2-h))}{1-\exp (-(q / 2-h))}\right) \\
& \leq \exp [-(p-1+q /(4 d))|\widetilde{\omega}|] \\
& \leq
\end{aligned}
$$

and (3.34) by

$$
\begin{aligned}
& 1+\frac{2 d \exp [-(p-2)(2 d u-2 u+2)]}{1-2 d \exp (-p)} \cdot \frac{\exp (-(q / 2-h))}{1-\exp (-(q / 2-h))} \\
& \leq 1+\frac{4 d \exp [-(p-2)(2 d u-2 u+2)]}{q / 2-h} \\
& \leq 1+\frac{16 d \exp [-(p-2)(2 d u-2 u+2)]}{q} \\
& \leq 1+q /(4 d) \\
& \leq \exp (q /(4 d)) .
\end{aligned}
$$

In $(3.51)-(3.53)$ we use the fact that, for any $\widetilde{\omega}$ with diam $\widetilde{\omega} \geq u$, the number of plaquettes in $\widetilde{\omega}$ is greater than or equal to $2 d u-2 u+2$. From (3.50) and (3.51) we get

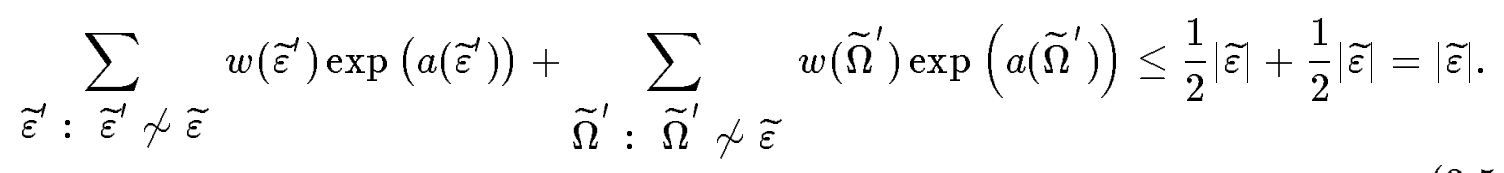


In a similar way

$$
\begin{aligned}
& \sum_{\tilde{\varepsilon}^{\prime}: \widetilde{\varepsilon}^{\prime} \psi \widetilde{\Omega}} w\left(\widetilde{\varepsilon}^{\prime}\right) \exp \left(a\left(\widetilde{\varepsilon}^{\prime}\right)\right)+\sum_{\widetilde{\Omega}^{\prime}: \widetilde{\Omega}^{\prime} \psi \widetilde{\Omega}} w\left(\tilde{\Omega}^{\prime}\right) \exp \left(a\left(\tilde{\Omega}^{\prime}\right)\right) \\
& \leq \sum_{\vec{\varepsilon}: \vec{\varepsilon}^{1} \cap \partial \widetilde{\Omega} \neq \emptyset} w\left(\widetilde{\varepsilon}^{\prime}\right) \exp \left(a\left(\vec{\varepsilon}^{\prime}\right)\right) \\
& +\sum_{\tilde{\omega}^{\prime}: \tilde{\omega}^{\prime} \cap \tilde{\Omega} \neq \emptyset \tilde{\Omega}^{\prime}: w_{0}\left(\tilde{\Omega}^{\prime}\right)=\widetilde{\omega}^{\prime}}\left|w\left(\tilde{\Omega}^{\prime}\right)\right| \exp \left(a\left(\tilde{\Omega}^{\prime}\right)\right) \\
& \leq|\partial \widetilde{\Omega}|+\sum_{\widetilde{\omega}^{\prime}: \tilde{\omega}^{\prime} \cap \widetilde{\Omega} \neq \emptyset} \exp \left(-(p-2)\left|\widetilde{\omega}^{\prime}\right|\right) \\
& \leq|\partial \widetilde{\Omega}|+|\widetilde{\Omega}| \frac{2 d \exp [-(p-2)(2 d u-2 u+2)]}{1-2 d \exp (-p)} \\
& \leq|\partial \widetilde{\Omega}|+4 d \exp [-(p-2)(2 d u-2 u+2)]|\widetilde{\Omega}| \\
& \leq|\partial \widetilde{\Omega}|+2 d \exp [-(p-2)(d u-u+1)]|\widetilde{\Omega}| \\
& =|\partial \widetilde{\Omega}|+h|\widetilde{\Omega}|,
\end{aligned}
$$

where

$$
|\partial \widetilde{\Omega}|=\sum_{i=0}^{n(\widetilde{\Omega})}\left|\widetilde{\omega}_{i}(\widetilde{\Omega})\right| .
$$

Contour model 3. Our next contour model describes a situation where one has a finite number of metastable phases, with a single stable phase that is dominating, owing to its more substantial low-energy excitations. The model contains contours of two types, long contours $\omega$ and short contours $\varepsilon$. Both long and short contours are triples, $\boldsymbol{\omega}=(\widetilde{\omega}, E(\boldsymbol{\omega}), I(\boldsymbol{\omega}))$ and $\varepsilon=(\widetilde{\varepsilon}, E(\varepsilon), I(\varepsilon))$, where

(i) $\tilde{\omega}$ and $\widetilde{\varepsilon}$ are sets of the plaquettes of the dual lattice $\widetilde{\mathbb{Z}}^{d}$ which are the boundaries of finite connected and simple-connected sets on $\mathbb{Z}^{d}$;

(ii) $(E(\boldsymbol{\omega}), I(\boldsymbol{\omega}))$ and $(E(\varepsilon), I(\varepsilon))$ are two pairs of distinct numbers from $\{0, \ldots$, $m\}$.

In fact, set $\{0, \ldots, m\}$ may be replaced by an arbitrary finite set. Physically, sets $\widetilde{\omega}$ and $\widetilde{\varepsilon}$ (called, as before, interfaces) separate different 'phases' (outside and inside $\widetilde{\omega}$ and $\widetilde{\varepsilon}$, respectively), and numbers $E(\cdot)$ and $I(\cdot)$ label these phases. As in model 2, we suppose that for any long contour $\omega$

$$
\operatorname{diam} \widetilde{\omega} \geq u>1
$$

while for short contours $\varepsilon$

$$
\operatorname{diam} \tilde{\varepsilon}<u \text {. }
$$

As in Section2, we denote by Int $\widetilde{\omega}$ and Int $\widetilde{\varepsilon}$ the subsets of $\mathbb{Z}^{d}$ enclosed by $\widetilde{\omega}$ and $\widetilde{\varepsilon}$, respectively, and by Ext $\widetilde{\omega}$ and Ext $\widetilde{\varepsilon}$ the complements $\mathbb{Z}^{d} \backslash$ Int $\widetilde{\omega}$ and $\mathbb{Z}^{d} \backslash$ Int $\widetilde{\varepsilon}$, respectively. 
The compatibility rules for collections of $\omega^{\prime}$ 's and $\varepsilon$ 's in model 3 are more complicated (and not even reduced to binary ones). We state these rules for any pair of long or short contours, from a given collection, which are not separated by any third long contour from the same collection. Here, we say that $\boldsymbol{\omega}^{\prime}=\left(\widetilde{\omega}^{\prime}, E^{\prime}, I^{\prime}\right)$ and $\boldsymbol{\omega}^{\prime \prime}=\left(\widetilde{\omega}^{\prime \prime}, E^{\prime \prime}, I^{\prime \prime}\right)$ are separated by $\boldsymbol{\omega}$ if either $\widetilde{\omega}^{\prime} \subset$ Int $\widetilde{\omega}, \widetilde{\omega}^{\prime \prime} \subset$ Ext $\widetilde{\omega}$ or $\widetilde{\omega}^{\prime \prime} \subset$ Int $\widetilde{\omega}, \widetilde{\omega}^{\prime} \subset \operatorname{Ext} \widetilde{\omega}$, and similarly for pairs $\varepsilon^{\prime}, \omega^{\prime \prime}$ and $\varepsilon^{\prime}, \varepsilon^{\prime \prime}$. Thus, two long contours, $\boldsymbol{\omega}^{\prime}=\left(\widetilde{\omega}^{\prime}, E^{\prime}, I^{\prime}\right)$ and $\boldsymbol{\omega}^{\prime \prime}=\left(\widetilde{\omega}^{\prime \prime}, E^{\prime \prime}, I^{\prime \prime}\right)$, not separated by a third long contour are compatible if

(i.1) $\tilde{\omega}^{\prime} \cap \widetilde{\omega}^{\prime \prime}=\emptyset$, Int $\widetilde{\omega}^{\prime} \subset$ Int $\widetilde{\omega}^{\prime \prime}$ and $I^{\prime \prime}=E^{\prime}$;

(i.2) $\tilde{\omega}^{\prime} \cap \widetilde{\omega}^{\prime \prime}=\emptyset$, Int $\widetilde{\omega}^{\prime \prime} \subset \operatorname{Int} \widetilde{\omega}^{\prime}$ and $I^{\prime}=E^{\prime \prime}$;

(i.3) $\tilde{\omega}^{\prime} \cap \widetilde{\omega}^{\prime \prime}=\emptyset$, Int $\widetilde{\omega}^{\prime} \cap \operatorname{Int} \widetilde{\omega}^{\prime \prime}=\emptyset$ and $E^{\prime \prime}=E^{\prime}$;

(i.4) $\widetilde{\omega}^{\prime} \cap \widetilde{\omega}^{\prime \prime} \neq \emptyset$, Int $\widetilde{\omega}^{\prime} \subset$ Int $\widetilde{\omega}^{\prime \prime}$ and either $E^{\prime \prime}>I^{\prime \prime}=E^{\prime}>I^{\prime}$ or $E^{\prime \prime}<I^{\prime \prime}=E^{\prime}<I^{\prime}$

(i.5) $\tilde{\omega}^{\prime} \cap \widetilde{\omega}^{\prime \prime} \neq \emptyset$, Int $\widetilde{\omega}^{\prime \prime} \subset$ Int $\widetilde{\omega}^{\prime}$ and either $E^{\prime}>I^{\prime}=E^{\prime \prime}>I^{\prime \prime}$ or $E^{\prime}<I^{\prime}=E^{\prime \prime}<I^{\prime \prime}$

(i.6) $\widetilde{\omega}^{\prime} \cap \widetilde{\omega}^{\prime \prime} \neq \emptyset$, Int $\widetilde{\omega}^{\prime} \cap$ Int $\widetilde{\omega}^{\prime \prime}=\emptyset$ and either $I^{\prime \prime}>E^{\prime \prime}=E^{\prime}>I^{\prime}$ or $I^{\prime \prime}<E^{\prime \prime}=E^{\prime}<I^{\prime}$.

Two short contours, $\varepsilon^{\prime}=\left(\widetilde{\varepsilon}^{\prime}, E^{\prime}, I^{\prime}\right)$ and $\varepsilon^{\prime \prime}=\left(\widetilde{\varepsilon}^{\prime \prime}, E^{\prime \prime}, I^{\prime \prime}\right)$, not separated by a long contour are compatible if

(ii.1) $\widetilde{\varepsilon}^{\prime} \cap \widetilde{\varepsilon}^{\prime \prime}=\emptyset$ and $E^{\prime}=E^{\prime \prime}$;

(ii.2) $\widetilde{\varepsilon}^{\prime} \cap \widetilde{\varepsilon}^{\prime \prime} \neq \emptyset$, Int $\widetilde{\varepsilon}^{\prime} \subset$ Int $\widetilde{\varepsilon}^{\prime \prime}$ or Int $\widetilde{\varepsilon}^{\prime \prime} \subset$ Int $\widetilde{\varepsilon}^{\prime}, E^{\prime}=E^{\prime \prime}$ and either $E^{\prime}>I^{\prime}, \mathbb{E}^{\prime \prime}>I^{\prime \prime}$ or $E^{\prime}<I^{\prime}, E^{\prime \prime}<I^{\prime \prime}$

(ii.3) $\widetilde{\varepsilon}^{-1} \cap \widetilde{\varepsilon}^{\prime \prime} \neq \emptyset$, Int $\vec{\varepsilon}^{\prime \prime} \cap \operatorname{Int} \vec{\varepsilon}=\emptyset, E^{\prime}=E^{\prime \prime}$ and either $E^{\prime}>I^{\prime}, E^{\prime \prime}<I^{\prime \prime}$ or $E^{\prime}<I^{\prime}, E^{\prime \prime}>I^{\prime \prime}$.

Finally, long contour $\omega^{\prime}=\left(\widetilde{\omega}^{\prime}, E^{\prime}, I^{\prime}\right) \quad \varepsilon^{\prime \prime}=\left(\widetilde{\varepsilon}^{\prime \prime}, E^{\prime \prime}, I^{\prime \prime}\right)$ not separated by another long contour are compatible if

(iii.1) $\widetilde{\omega}^{\prime} \cap \widetilde{\varepsilon}^{\prime \prime}=\emptyset$, Int $\widetilde{\varepsilon}^{\prime \prime} \subset$ Int $\widetilde{\omega}^{\prime}$ and $I^{\prime}=E^{\prime \prime}$;

(iii.2) $\widetilde{\omega}^{\prime} \cap \widetilde{\varepsilon}^{\prime \prime}=\emptyset$, Int $\widetilde{\omega}^{\prime} \cap \operatorname{Int} \widetilde{\varepsilon}^{\prime \prime}=\emptyset$ and $E^{\prime \prime}=E^{\prime}$;

(iii.3) $\widetilde{\omega}^{\prime} \cap \widetilde{\varepsilon}^{\prime \prime} \neq \emptyset$, Int $\widetilde{\varepsilon}^{\prime \prime} \subset$ Int $\widetilde{\omega}^{\prime}$ and either $E^{\prime}>I^{\prime}=E^{\prime \prime}>I^{\prime \prime}$ or $E^{\prime}<I^{\prime}=E^{\prime \prime}<I^{\prime \prime}$

(iii.4) $\widetilde{\omega}^{\prime} \cap \widetilde{\varepsilon}^{\prime \prime} \neq \emptyset$, Int $\widetilde{\omega}^{\prime} \cap \operatorname{Int} \widetilde{\varepsilon}^{\prime \prime}=\emptyset$ and either $I^{\prime \prime}>E^{\prime \prime}=E^{\prime}>I^{\prime}$ or $I^{\prime \prime}<E^{\prime \prime}=E^{\prime}<I^{\prime}$.

Note that the situation Int $\widetilde{\omega} \subset$ Int $\widetilde{\varepsilon}$ is impossible, and the inclusion $\subset$ is always understood in the strict sense.

We say that a collection $\left\{\boldsymbol{\varepsilon}_{i}, \boldsymbol{\omega}_{j}\right\}$ of short and long contours is compatible if any two of the contours not separated by a third long contour from the collection are compatible. The motivation of such a definition of compatibility is as follows. We give the definition in such a way that any compatible collection of long contours $\left\{\boldsymbol{\omega}_{j}\right\}$ uniquely defines a configuration $X=X\left(\left\{\boldsymbol{\omega}_{j}\right\}\right): x \in \mathbb{Z}^{d} \mapsto X_{x} \in\{0,1, \ldots, m\}$, with every $\widetilde{\omega}_{j}$ separating the region with $X_{x}=E\left(\omega_{j}\right)$ from the region with $X_{x}=I\left(\omega_{j}\right)$. The short contours $\varepsilon_{i}$, with $E\left(\varepsilon_{i}\right)=k$, are situated inside the set $\left\{x \in \mathbb{Z}^{d} \mid X_{x}=k\right\}$; they do not change the configuration defined by $\left\{\boldsymbol{\omega}_{j}\right\}$.

In the current model, we suppose that the statistical weights of long and short contours satisfy the bounds

$$
|w(\boldsymbol{\omega})| \leq \exp [-p|\widetilde{\omega}| \cdot|E(\boldsymbol{\omega})-I(\boldsymbol{\omega})|]
$$


and

$$
|w(\varepsilon)| \leq \exp [-p|\widetilde{\varepsilon}| \cdot|E(\varepsilon)-I(\varepsilon)|] .
$$

This means that the statistical weights of the contours depend not only on the geometry of their interfaces but also on their labels $E(\cdot)$ and $I(\cdot)$. For every value $k=0, \ldots, m$, the metastable partition function in $V$, with the external phase $k$, is defined as

$$
Z^{(k)}(V)=\sum_{\left\{\varepsilon_{i}\right\}:\left\{\widetilde{\varepsilon}_{i}\right\} \subseteq V, i} \prod_{i} w\left(\widetilde{\varepsilon}_{i}\right)
$$

Here, the summation is extended to the compatible collections $\left\{\varepsilon_{i}\right\}$ where each $\widetilde{\varepsilon}_{j}$ is contained in $V$ in the $\mathbb{R}^{d}$-sense, and $E\left(\varepsilon_{j}\right) \equiv k$. Further, we suppose that $m$ is even and phase $\frac{m}{2}$ is the dominant one, in the sense that

$$
\frac{Z^{(k)}(V)}{Z^{(m / 2)}(V)} \leq \exp [-|V| 8 d \exp (-(p-2)(d u-u+1))] .
$$

The full partition function of the contour model 3 is

$$
\begin{aligned}
& \Xi^{(k)}(V)= \sum_{\left\{\varepsilon_{i}, \omega_{j}\right\}:\left\{\widetilde{\varepsilon}_{i}, \widetilde{\omega}_{j}\right\} \subseteq V, i} \prod_{i} w\left(\varepsilon_{i}\right) \prod_{j} w\left(\omega_{j}\right) . \\
& E\left(\varepsilon_{i}^{\text {ext }}\right)=E\left(\boldsymbol{\omega}_{j}^{e x t}\right)=k
\end{aligned}
$$

Here, the summation is extended to the compatible collections $\left\{\boldsymbol{\varepsilon}_{i}, \boldsymbol{\omega}_{j}\right\}$ such that each $\widetilde{\varepsilon}_{i}$ and $\widetilde{\omega}_{j}$ is contained in $V$ in the $\mathbb{R}^{d}$-sense and $E\left(\varepsilon_{i}^{e x t}\right) \equiv E\left(\omega_{j}^{e x t}\right) \equiv k$. Furthermore, $\varepsilon_{i}^{e x t}$ and $\omega_{j}^{e x t}$ are the external (i.e. not enclosed by another one) long and short contours, respectively. We are interested in constructing a polymer expansion for the logarithm of the partition function $\Xi^{(m / 2)}(V)$ corresponding to the dominant phase. To simplify the notation, we set below $Z(V)=\Xi^{(m / 2)}(V)$.

It is convenient to construct, in the current model, an analogue of the bulky contour from models 1 and 2 . This is a compatible collection, $\Omega=\left\{\boldsymbol{\omega}_{j}\right\}$, of long contours, with $E\left(\left\{\boldsymbol{\omega}_{j}\right\}\right)=\frac{m}{2}$, such that the set $\widetilde{\Omega}=\tilde{\Omega}\left(\left\{\boldsymbol{\omega}_{j}\right\}\right)$ given by

$$
\widetilde{\Omega}=\left\{x \in \mathbb{Z}^{d} \mid X_{x}\left(\left\{\omega_{j}\right\}\right) \neq \frac{m}{2}\right\}
$$

is connected. Denote by $\partial_{r} \Omega$ the long contour from collection $\left\{\boldsymbol{\omega}_{j}(\Omega)\right\}$ whose interface is $\partial_{r} \widetilde{\Omega}$. As before, we denote by $\partial_{0} \Omega$ the unique external long contour in collection $\left\{\partial_{r} \Omega\right\}$. By construction, $E\left(\partial_{0} \Omega\right)=I\left(\partial_{r} \Omega\right)=\frac{m}{2}, r \neq 0$ while $E\left(\omega_{j}(\Omega)\right), I\left(\omega_{j}(\Omega)\right) \neq$ $\frac{m}{2}$ for $\boldsymbol{\omega}_{j}(\Omega)$ from $\left\{\boldsymbol{\omega}_{j}(\Omega)\right\} \backslash\left\{\partial_{r} \Omega\right\}$. The collection $\left\{\boldsymbol{\omega}_{j}(\Omega)\right\} \backslash\left\{\partial_{r} \Omega\right\}$ partitions $\tilde{\Omega}$ into subsets $\tilde{\Omega}^{(k)}, k \neq \frac{m}{2}$ with $X_{x}(\Omega)=k$ when $x \in \tilde{\Omega}^{(k)}$.

Every compatible collection $\left\{\varepsilon_{i}, \omega_{j}\right\}$ can now be treated as a compatible collection $\left\{\varepsilon_{i}, \Omega_{n}\right\}$, and $(3.63)$ can be rewritten as

$$
Z(V)=\sum_{\left\{\varepsilon_{i}, \Omega_{n}\right\}:} \prod_{\left\{\widetilde{\varepsilon}_{i}, \tilde{\Omega}_{n}\right\} \subseteq V} w\left(\varepsilon_{i}\right) \prod_{n} \prod_{j} w\left(\omega_{j}\left(\Omega_{n}\right)\right)
$$




$$
\begin{gathered}
=\sum_{\left\{\Omega_{n}\right\}:\left\{\widetilde{\Omega}_{n}\right\} \subseteq V} Z^{(m / 2)}\left(V \backslash\left(\cup_{n} \widetilde{\Omega}_{n}\right) \mid\left\{\partial_{r} \Omega_{n}\right\}\right) \\
\times \prod_{n} \prod_{j} w\left(\boldsymbol{\omega}_{j}\left(\Omega_{n}\right)\right) \prod_{k} Z^{(k)}\left(\widetilde{\Omega}_{n}^{(k)} \mid \Omega_{n}\right) .
\end{gathered}
$$

Here the conditioned metastable partition functions are defined as follows:

$$
\begin{aligned}
& Z^{(m / 2)}\left(V \backslash\left(\cup_{n} \widetilde{\Omega}_{n}\right) \mid\left\{\partial_{r} \Omega_{n}\right\}\right) \\
& =\sum_{\left\{\varepsilon_{i}\right\}:\left\{\tilde{\varepsilon}_{i}\right\} \subseteq V \backslash\left(\cup_{n} \widetilde{\Omega}_{n}\right), E\left(\varepsilon_{i}\right)=m / 2,} \prod_{i} w\left(\varepsilon_{i}\right) \\
& \text { any pair } \varepsilon_{i}, \partial_{r} \Omega_{n} \text { is compatible }
\end{aligned}
$$

and

$$
Z^{(k)}\left(\widetilde{\Omega}_{n}^{(k)} \mid \Omega_{n}\right)=\sum_{\substack{\left\{\varepsilon_{i}\right\}:\left\{\tilde{\varepsilon}_{i}\right\} \subseteq \widetilde{\Omega}_{n}^{(k)}, E\left(\varepsilon_{i}\right)=k, \\ \text { any pair } \varepsilon_{i}, \omega_{j}\left(\Omega_{n}\right) \text { is compatible }}} \prod_{i} w\left(\varepsilon_{i}\right),
$$

cf. (3.61). The meaning of the notation $\left\{\widetilde{\varepsilon}_{i}, \widetilde{\Omega}_{n}\right\} \subseteq V,\left\{\widetilde{\Omega}_{n}\right\} \subseteq V$, etc, is the same as in model 2.

Given a bulky contour $\Omega \subset \mathbb{Z}^{d}$ we define

$$
w(\Omega)=\prod_{j} w\left(\boldsymbol{\omega}_{j}(\Omega)\right) \prod_{k} \frac{Z^{(k)}\left(\tilde{\Omega}^{(k)} \mid \Omega\right)}{Z^{(m / 2)}\left(\tilde{\Omega}^{(k)} \mid \Omega^{*}\right)} .
$$

Here the collection $\Omega^{*}=\left\{\boldsymbol{\omega}_{j}^{*}\right\}$ is obtained from $\Omega=\left\{\boldsymbol{\omega}_{j}\right\}$ by setting $\widetilde{\omega}_{j}^{*}=\widetilde{\omega}_{j}$, $I\left(\boldsymbol{\omega}_{j}^{*}\right)=\frac{m}{2}$ and $E\left(\boldsymbol{\omega}_{j}^{*}\right)=E\left(\boldsymbol{\omega}_{j}\right)+\frac{m}{2}-I\left(\boldsymbol{\omega}_{j}\right)$. Then (3.65) can be rewritten as

$$
\begin{aligned}
Z(V)= & \sum_{\left\{\boldsymbol{\varepsilon}_{i}, \Omega_{n}\right\}:\left\{\widetilde{\varepsilon}_{i}, \tilde{\Omega}_{n}\right\} \subseteq V} \prod_{i} w\left(\varepsilon_{i}\right) \prod_{n} \prod_{j} w\left(\boldsymbol{\omega}_{j}\left(\Omega_{n}\right)\right) \\
= & \sum_{\left\{\Omega_{n}\right\}:} Z^{(m / 2)}\left(V \backslash\left(\tilde{\Omega}_{n}\right\} \subseteq V\right. \\
& \left.\left.\times \prod_{n} w\left(\Omega_{n}\right) \prod_{k}\right) \mid\left\{\partial_{r} \Omega_{n}\right\}\right)
\end{aligned}
$$

and expanding $Z^{(m / 2)}(\cdot)$ in form (3.66) and (3.67) yields the representation

$$
Z(V)=\sum_{\left\{\varepsilon_{i}, \Omega_{n}\right\}:\left\{\widetilde{\varepsilon}_{i}, \widetilde{\Omega}_{n}\right\} \subseteq V} \prod_{i} w\left(\varepsilon_{i}\right) \prod_{n} w\left(\Omega_{n}\right)
$$


that is an analogue of (3.48). Here we suppose that $E\left(\varepsilon_{i}\right)=\frac{m}{2}$ for all $i$. The compatibility rule for the $\varepsilon$ 's and $\Omega$ 's is an appropriate analogue of that in (3.48). More precisely, the rule is the binary one and it requires for any pair $\left(\Omega, \Omega^{\prime}\right),\left(\varepsilon, \varepsilon^{\prime}\right)$ and $(\Omega, \varepsilon)$ :

(i) $\widetilde{\Omega} \cap \widetilde{\Omega}^{\prime}=\emptyset$;

(ii) $\tilde{\varepsilon} \cap \widetilde{\varepsilon}=\emptyset$;

(iii) either

(iii.1) Int $\widetilde{\varepsilon} \subset \widetilde{\Omega}^{c}$ and, for any $r$, the long contour $\partial_{r} \Omega$ and short contour $\varepsilon$ are compatible,

or

(iii.2) Int $\tilde{\varepsilon} \subset \widetilde{\Omega}$ and, for any $j$, a long contour $\left(\widetilde{\omega}_{j}(\Omega), E\left(\omega_{j}(\Omega)\right)+\frac{m}{2}-\right.$ $\left.I\left(\omega_{j}(\Omega)\right), \frac{m}{2}\right)$ constructed from $\omega_{j}(\Omega)=\left(\widetilde{\omega}_{j}(\Omega), E\left(\omega_{j}(\Omega)\right), I\left(\omega_{j}(\Omega)\right),\right)$, and short contour $\varepsilon$ are compatible.

Proposition 3.5 Under conditions (3.59), (3.60) and (3.62), with

$$
p \geq 4+\log (2 d)
$$

the logarithm of partition function (3.63), with $k=m / 2$, (or equivalently, the logarithm of partition function (3.70)) admits a polymer expansion of form (3.3), where properties (3.6), (3.7) are valid with

$$
\begin{aligned}
a(\varepsilon) & =|\widetilde{\varepsilon}| \\
a(\Omega) & =\sum_{j}\left|\widetilde{\omega}_{j}(\Omega)\right|+h|\widetilde{\Omega}| .
\end{aligned}
$$

and $h=d \exp (-(p-2)(d u-u+1))$.

Proof. We proceed in a way similar to Propositions 3.2 and 3.4. In analogy with $(2.13)$ we define

$$
\|\varepsilon\|=|\widetilde{\varepsilon}| \cdot|E(\varepsilon)-I(\varepsilon)|,|| \boldsymbol{\omega}||=|\widetilde{\omega}| \cdot|E(\boldsymbol{\omega})-I(\boldsymbol{\omega})|
$$

A minor difference with the proof of the above propositions is caused by the fact that, in all calculations, $|\cdot|$ should be replaced by $\|\cdot\|$, and an additional summation over $|E(\cdot)-I(\cdot)|$ is carried on. This does not change the final bounds.

Another difference is that, instead of (3.22), we now have, in view of (3.62), the bound

$$
|w(\Omega)| \leq \exp \left(-p \sum_{j}|| \boldsymbol{\omega}_{j}(\Omega) \|-q^{\prime} \sum_{k}\left|\left[\tilde{\Omega}^{(k)}\right]\right|\right),
$$

where $q^{\prime}=8 d \exp (-(p-2)(d u-u+1))$.

Lemma 3.6 For $q=4 d \exp (-(p-2)(d u-u+1))$, the following bound holds true:

$$
\sum_{\Omega:\left\{\partial_{r} \Omega\right\} \text { is fixed }}|w(\Omega)| \leq \exp \left(-(p-1) \sum_{r}|| \partial_{r} \Omega||-q|\widetilde{\Omega}|\right) .
$$


Proof. The sum in the left-hand side of (3.75) is, in fact, over all possibilities to complete $\left\{\partial_{r} \Omega\right\}$ to $\left\{\boldsymbol{\omega}_{j}(\Omega)\right\}$. It is

$$
\begin{aligned}
& \leq\left(1+\sum_{\boldsymbol{\omega}: \operatorname{Int} \widetilde{\omega} \ni 0} \exp (-p|| \boldsymbol{\omega}||)\right)^{|\widetilde{\Omega}|} \\
& \leq\left(1+\sum_{\left.\widetilde{\omega}: \operatorname{Int} \widetilde{\omega} \ni 0 \sum_{I=0}^{m} \exp (-p|\omega| \cdot|E-I|)\right)^{|\widetilde{\Omega}|}}\right. \\
& \leq\left(1+4 \sum_{\tilde{\omega}: \operatorname{Int} \widetilde{\omega} \ni 0} \exp (-p|\omega|)\right)^{|\widetilde{\Omega}|} \\
& \leq\left(1+\frac{8 d \exp (-p(2 d u-2 u+2))}{1-2 d \exp (-p)}\right)^{|\widetilde{\Omega}|} \\
& \leq \exp [|\widetilde{\Omega}| 4 d \exp (-(p-2)(d u-u+1))]
\end{aligned}
$$

Combining (3.76) with (3.74), we obtain the lemma.

$$
\text { Now (3.22) is valid for } \sum_{\Omega:\left\{\partial_{r} \Omega\right\} \text { is fixed }}|w(\Omega)| \text {, and we can proceed further }
$$

exactly as in Propositions 3.2 and 3.4 .

Usually, a 'physical' model can be described directly in terms of a contour model only when it possesses a unique limiting Gibbs measure. In fact, in many cases the description by means of contour model is a natural way to prove uniqueness. To analyse physical models with more than one phase, one usually needs the rather involved machinery of Pirogov-Sinai Theory. A situation where it can be avoided arises in models with two symmetric dominant phases. The simplest example is the symmetry between + and - phases of the Ising model with zero external magnetic field. Our next contour model provides a description of such situations.

Contour model 4. The setting is similar to model 3, with the following addenda reflecting the symmetry between two selected phases, say $\frac{m}{2}-\frac{1}{2}$ and $\frac{m}{2}+\frac{1}{2}$ (the value $m$ is now supposed to be odd):

(i) there exists a permutation, $\nu$, of the set $\{0,1, \ldots, m\}$, such that $\frac{m}{2}+\frac{1}{2}=$ $\nu\left(\frac{m}{2}-\frac{1}{2}\right)$ and every long contour $\boldsymbol{\omega}$ with $E(\boldsymbol{\omega})=k$ and $I(\boldsymbol{\omega})=l$ has the same statistical weight as a similar long contour with $E(\boldsymbol{\omega})=\nu(k), I(\boldsymbol{\omega})=\nu(l)$; every short contour $\varepsilon$ with $E(\varepsilon)=k$ and $I(\varepsilon)=l$ has the same statistical weight as an analogous short contour with $E(\varepsilon)=\nu(k), I(\varepsilon)=\nu(l)$;

(ii) phases $\frac{m}{2}-\frac{1}{2}$ and $\frac{m}{2}+\frac{1}{2}$ are the dominant ones, in the sense that

$$
Z^{(m / 2-1 / 2)}(V)=Z^{(m / 2+1 / 2)}(V)
$$


and for $k \neq \frac{m}{2} \pm \frac{1}{2}$

$$
\frac{Z^{(k)}(V)}{Z^{(m / 2-1 / 2)}(V)} \leq \exp [-|V| 16 d \exp (-(p-2)(d u-u+1))] \text {. }
$$

Like (3.63), the full partition functions in $V$ are defined as

$$
\begin{aligned}
& \Xi^{(k)}(V)=\sum_{\left\{\boldsymbol{\varepsilon}_{i}, \boldsymbol{\omega}_{j}\right\}:\left\{\widetilde{\varepsilon}_{i}, \widetilde{\omega}_{j}\right\} \subseteq V:} \prod_{i} w\left(\varepsilon_{i}\right) \prod_{j} w\left(\boldsymbol{\omega}_{j}\right), \\
& E\left(\varepsilon_{i}^{e x t}\right)=E\left(\omega_{j}^{e x t}\right)=k
\end{aligned}
$$

where the range of summation is the same as in (3.63). The symmetry immediately gives that

$$
Z^{(m / 2-1 / 2)}(V)=Z^{(m / 2+1 / 2)}(V), \Xi^{(m / 2-1 / 2)}(V)=\Xi^{(m / 2+1 / 2)}(V) .
$$

Proposition 3.7 Under conditions (3.59), (3.60) and (3.77), with $p$ satisfying condition (3.71), the logarithm of partition function (3.78), with $k=\frac{m}{2} \pm \frac{1}{2}$, admits a polymer expansion of form (3.3), with a defined as in Proposition 3.5.

Proof. Owing to symmetry between phases $\frac{m}{2}-\frac{1}{2}$ and $\frac{m}{2}+\frac{1}{2}$, one can consider long contours $\boldsymbol{\omega}$ with $E(\boldsymbol{\omega})=\frac{m}{2}-\frac{1}{2}$ and $I(\boldsymbol{\omega})=\frac{m}{2}+\frac{1}{2}$ as not changing the phase (i.e. one can put $E(\boldsymbol{\omega})=I(\boldsymbol{\omega})=\frac{m}{2}-\frac{1}{2}$ keeping the partition functions $\Xi^{(m / 2-1 / 2)}(V)$ unchanged). This allows us to include such contours in the class of short contours of phase $\frac{m}{2}-\frac{1}{2}$. We still have, for $k \neq \frac{m}{2} \pm \frac{1}{2}$,

$$
\frac{Z^{(k)}(V)}{Z^{(m / 2-1 / 2)}(V)} \leq \exp [-|V| 8 d \exp (-(p-2)(d u-u+1))]
$$

because the new short contours can add, to the exponent in the right-hand side of (3.79), the correction of the order $|V| d \exp (-p(2 d u-2 u+2))$ at most. Thus, Proposition 3.7 is reduced to Proposition 3.5.

Our final models (5 and 6 ) have a more complicated geometry of long and short contours. We do not have an obvious explanation of the origin of this geometry, apart from the fact that it covers the case of the quantum system under consideration.

Contour model 5. We first consider the case of a unique dominant phase. Model 5 , like models 3 and 4, contains long and short contours. A long contour is now denoted by $\zeta$ and defined as a pair $\left(\left\{\boldsymbol{\omega}_{j}\right\}, \sigma\right)$. Here,

(i) each $\boldsymbol{\omega}_{j}=\boldsymbol{\omega}_{j}(\boldsymbol{\zeta})$ is a contour (either long or short) from the previous model, and $\left\{\boldsymbol{\omega}_{j}\right\}$ is a finite, non-empty compatible collection, again in the sense of the previous model;

(ii) $\sigma=\sigma(\zeta)$ is a finite set of plaquettes of dual lattice $\widetilde{\mathbb{Z}}^{d}$; 
(iii) the union $\left(\cup_{j} \widetilde{\omega}_{j}(\zeta)\right) \cup \sigma(\zeta)$ denoted below by $\widetilde{\zeta}=\widetilde{\zeta}(\zeta)$ is a connected set of plaquettes of $\widetilde{\mathbb{Z}}^{d}$;

(iv) for at least one $\omega_{j}(\zeta)$,

$$
\operatorname{diam} \widetilde{\omega}_{j}(\zeta) \geq u
$$

A short contour is denoted, as before, by $\varepsilon$ and defined again as a pair $\left(\left\{\boldsymbol{\omega}_{j}\right\}, \sigma\right)$ (or $\left.\left(\left\{\boldsymbol{\omega}_{j}(\varepsilon)\right\}, \sigma(\varepsilon)\right)\right)$ for which the above conditions (i) - (iii) are valid, but (iv) is not:

$\left(\right.$ iv' $\left.^{\prime}\right)$ for each $\omega_{j}(\varepsilon)$,

$$
\operatorname{diam} \widetilde{\omega}_{j}(\varepsilon)<u
$$

The union $\left(\cup_{j} \widetilde{\omega}_{j}(\varepsilon)\right) \cup \sigma(\varepsilon)$ is denoted by $\widetilde{\varepsilon}=\widetilde{\varepsilon}(\varepsilon)$.

A collection $\left\{\varepsilon_{i}, \zeta_{j}\right\}$ of short and long contours is now called compatible if

(i) the $\widetilde{\varepsilon}_{i}$ 's and $\widetilde{\zeta}_{j}$ 's form a collection of pairwise non-intersecting subsets of $\widetilde{\mathbb{Z}}^{d}$;

(ii) the whole collection of the $\omega_{s}\left(\varepsilon_{i}\right)$ 's and $\omega_{t}\left(\zeta_{j}\right)$ 's from contours $\varepsilon_{i}$ and $\zeta_{j}$ is compatible, in the sense of model 4.

We suppose, in the current model, that the statistical weights of long and short contours satisfy the bounds

$$
|w(\zeta)| \leq \exp \left(-p \sum_{i}\left|\tilde{\boldsymbol{\omega}}_{i}(\boldsymbol{\zeta})\right| \cdot\left|E\left(\boldsymbol{\omega}_{i}(\boldsymbol{\zeta})\right)-I\left(\boldsymbol{\omega}_{i}(\boldsymbol{\zeta})\right)\right|-t|\sigma(\zeta)|\right)
$$

and

$$
|w(\varepsilon)| \leq \exp \left[-p\left|\widetilde{\omega}_{i}(\varepsilon)\right| \cdot\left|E\left(\boldsymbol{\omega}_{i}(\varepsilon)\right)-I\left(\boldsymbol{\omega}_{i}(\varepsilon)\right)\right|-t|\sigma(\varepsilon)|\right] .
$$

As in the previous models, we introduce the metastable partition functions

$$
Z^{(k)}(V)=\sum_{\left\{\varepsilon_{j}\right\}:\left\{\widetilde{\varepsilon}_{j}\right\} \subseteq V, \prod_{j}} w\left(\varepsilon_{j}\right)
$$

with the range of summation similar to (3.61). We also assume that $Z^{(m / 2)}(V)$ is dominating in the sense that

$$
\frac{Z^{(k)}(V)}{Z^{(m / 2)}(V)} \leq \exp \left[-\left|[V]_{v}\right| 8 d \exp (-(p-2 \log (2 d)-2)(d u-u+1))\right]
$$

for each $k=0, \ldots, m$ with $k \neq m / 2$ and $[V]_{v}=\left\{x \in V \mid \operatorname{dist}\left(x, V^{c}\right) \geq v+1\right\}$ where $v \geq 1$ is a constant.

The full partition functions are defined as

$$
\Xi^{(k)}(V)=\sum_{\substack{\left\{\varepsilon_{i}, \zeta_{j}\right\}:\left\{\widetilde{\varepsilon}_{i}, \tilde{\zeta}_{j}\right\} \subseteq V, E\left(\varepsilon_{i}^{e x t}\right)=E\left(\boldsymbol{\omega}_{n}^{e x t}\left(\boldsymbol{\zeta}_{j}\right)\right)=k}} \prod_{i} w\left(\varepsilon_{i}\right) \prod_{j} w\left(\zeta_{j}\right) .
$$

Like (3.63), the summation in (3.87) is extended to the compatible collections $\left\{\varepsilon_{i}, \boldsymbol{\zeta}_{j}\right\}$ such that each $\tilde{\varepsilon}_{i}$ and $\tilde{\zeta}_{j}$ is contained in $V$ in the $\mathbb{R}^{d}$-sense and $E\left(\varepsilon_{i}^{e x t}\right) \equiv$ $E\left(\zeta_{j}^{e x t}\right) \equiv k$. 
As before, it is convenient to introduce an analogue of a bulky contour. It is again a compatible collection, $\Omega=\left\{\boldsymbol{\zeta}_{i}\right\}$, of long contours, with $E\left(\left\{\boldsymbol{\zeta}_{i}\right\}\right)=E\left(\left\{\boldsymbol{\omega}_{j}\left(\boldsymbol{\zeta}_{i}\right)\right\}\right)=\frac{m}{2}$, such that the set $\widetilde{\Omega}=\widetilde{\Omega}\left(\left\{\zeta_{i}\right\}\right)$ given by

$$
\widetilde{\Omega}=\left\{x \in \mathbb{Z}^{d} \mid X\left(\left\{\zeta_{i}\right\}\right) \neq \frac{m}{2}\right\}
$$

has the following property. The union of the boundary of $\widetilde{\Omega}$ with $\cup_{i} \sigma\left(\zeta_{i}\right)$ forms a connected set of plaquettes of $\widetilde{\mathbb{Z}}^{d}$. As before, $X\left(\left\{\boldsymbol{\zeta}_{i}\right\}\right)=X\left(\boldsymbol{\omega}_{j}\left(\left\{\boldsymbol{\zeta}_{i}\right\}\right)\right)$ is a configuration uniquely determined by the compatible collection $\left\{\boldsymbol{\zeta}_{i}\right\}$.

Repeating the construction from the previous models (see $(3.65)-(3.70)$ ), replacing the $\boldsymbol{\omega}_{j}$ 's (the long contours in the previous models) by the $\boldsymbol{\zeta}_{i}$ 's, one can write, for $Z(V)=\Xi^{(m / 2)}(V)$, a formula

$$
Z(V)=\sum_{\left\{\varepsilon_{i}, \Omega_{n}\right\} \subseteq V} \prod_{i} w\left(\varepsilon_{i}\right) \prod_{n} w\left(\Omega_{n}\right),
$$

identical to $(3.70)$.

Proposition 3.8 Under conditions (3.83) - (3.86), with

$$
p \geq 4+2 \log (2 d)+\frac{4 \log 2+\log v}{d u-u+1}, t \geq 2 \log (2 d)+1
$$

the logarithm of partition function (3.87), with $k=m / 2$, (or equivalently, the logarithm of partition function (3.89)) admits a polymer expansion of form (3.3), with

$$
\begin{aligned}
& a(\varepsilon)=|\widetilde{\varepsilon}|=|\sigma(\varepsilon)|+\sum_{j}\left|\widetilde{\omega}_{j}(\varepsilon)\right| \\
& a(\Omega)=\sum_{i}\left(\sum_{j}\left|\widetilde{\omega}_{j}\left(\zeta_{i}(\Omega)\right)\right|+\left|\sigma\left(\zeta_{i}(\Omega)\right)\right|\right)+h|\widetilde{\Omega}|,
\end{aligned}
$$

and $h=d \exp (-(p-2 \log (2 d)-2)(d u-u+1))$.

Proof. The idea of the proof is to repeat the proof of Proposition 3.5, with corresponding modifications. We put

$$
q=4 d \exp [-(p-2 \log (2 d)-2)(d u-u+1)]
$$

and substitute $p-2 \log (2 d)-2$ in place of $(p-2)$ in all corresponding calculations. To make it possible, one needs a summability of statistical weights $w(\zeta)$.

Lemma 3.9 For $p \geq 4+2 \log (2 d)$ and $t \geq 2 \log (2 d)$

$$
\sum_{\zeta: \cup_{j} \tilde{\omega}_{j}(\zeta) \cup \sigma(\zeta) \ni 0}|w(\zeta)| \leq \exp [-(p-2 \log (2 d))(2 d u-2 u+2)] .
$$


Proof. As $\cup_{j} \widetilde{\omega}_{j}(\zeta) \cup \sigma(\zeta)$ is connected and, at least for one value of $j$, the diameter of $\widetilde{\omega}_{j}(\zeta)$ is greater than or equal $u$, we have

$$
\begin{aligned}
& \sum_{\zeta: \cup_{j} \tilde{\omega}_{j}(\zeta) \cup \sigma(\zeta) \ni 0}|w(\zeta)| \\
& \leq \sum_{\zeta: \cup_{j} \tilde{\omega}_{j}(\zeta) \cup \sigma(\zeta) \ni 0} \sum_{I=-m}^{m} \exp \left(-p \sum_{j}\left|\widetilde{\omega}_{j}(\zeta)\right|\right. \\
& \left.\times\left|E\left(\boldsymbol{\omega}_{j}(\boldsymbol{\zeta})\right)-\left(I+E\left(\boldsymbol{\omega}_{j}(\boldsymbol{\zeta})\right)\right)\right|-t|\sigma(\zeta)|\right) \\
& \leq 4 \sum_{\zeta: \cup_{j} \widetilde{\omega}_{j}(\zeta) \cup \sigma(\zeta) \ni 0} \exp \left(-p \sum_{j}\left|\widetilde{\omega}_{j}(\zeta)\right|-t|\sigma(\zeta)|\right) \\
& \leq 4 \exp [-(p-t)(2 d u-2 u+2)] \\
& \times \sum_{\zeta: \cup_{j} \widetilde{\omega}_{j}(\zeta) \cup \sigma(\zeta) \ni 0} \exp \left(-t \sum_{j}\left|\widetilde{\omega}_{j}\right|-t|\sigma(\zeta)|\right) \\
& \leq 4 \exp [-(p-t)(2 d u-2 u+2)] \frac{2 d \exp (-t)}{1-2 d \exp (-t)} \\
& \leq \exp [-(p-2 \log (2 d))(2 d u-2 u+2)] \text {. }
\end{aligned}
$$

The proof of Proposition 3.8 can now proceed along the same line of arguments as that of Proposition 3.5. The only place where a modification is needed is in an analogue of bound (3.74). Because of the difference between (3.62) and (3.86), the analogue of (3.74) takes the form

$$
|w(\Omega)| \leq \exp \left(-\sum_{i}\left(p \sum_{j}|| \boldsymbol{\omega}_{j}\left(\zeta_{i}(\Omega)\right) \|+t\left|\sigma\left(\zeta_{i}(\Omega)\right)\right|\right)-q^{\prime} \sum_{k}\left|\left[\tilde{\Omega}^{(k)}\right]_{v}\right|\right),
$$

where $q^{\prime}=8 d \exp [-(p-2 \log (2 d)-2)(d u-u+1)]$ and $w(\Omega)$ is defined similarly to $(3.68)$.

$$
\begin{gathered}
\text { Obviously }\left|\tilde{\Omega} \backslash\left(\cup_{k}\left[\tilde{\Omega}^{(k)}\right]_{v}\right)\right| \text { is less than } 2 v \sum_{i} \sum_{j}\left|\widetilde{\omega}_{j}\left(\zeta_{i}(\Omega)\right)\right|, \text { and } \\
1 \geq 16 v d \exp (-(p-2 \log (2 d)-2)(d u-u+1))
\end{gathered}
$$

for $p \geq 4+2 \log (2 d)+\frac{4 \log 2+\log v}{d u-d+1}$. Hence

$$
|w(\Omega)| \leq \exp \left(-\sum_{i}\left((p-1) \sum_{j}|| \omega_{j}\left(\zeta_{i}(\Omega)\right)||+t\left|\sigma\left(\zeta_{i}(\Omega)\right)\right|\right)-q^{\prime} \sum_{k}\left|\widetilde{\Omega}^{(k)}\right|\right)
$$


With this remark, one can proceed further exactly as in the proof of Proposition 3.5.

Contour model 6 . The last contour model of this section describes a situation with two symmetric dominant phases, in the setup of model 5. Here, an analogue of Proposition 3.7 may be proved, which we refer to as Proposition 3.10, without stating it in an explicit form.

\section{Proof of Theorems 1 and 2}

In this section we use Propositions 3.8 and 3.10 in a special case of the ensemble of quantum contours (more precisely, of compatible collections of quantum contours) determined by (2.15), (2.16). As was noted before, our aim is to construct a polymer expansion for $\log \Xi^{(l)}(V)$ with $l=\frac{m}{2}$ for $m$ even and $l=\frac{m}{2} \pm \frac{1}{2}$ for $m$ odd, and obtain thereby the existence (and ergodicity) of states $\psi^{0}$ and $\psi^{ \pm}$figuring in Theorem 1. The remainig statements of Theorem 1 require an additional construction provided at the end of the section. The term probability is used below in the sense of the ensemble of quantum contours.

To match the notation from Section 3 we set, for a quantum contour $\zeta$ :

$$
\widetilde{\omega}_{j}(\zeta)=\widetilde{\gamma}_{j}^{0}(\zeta), \quad j=1, \ldots, n_{0}(\zeta)
$$

and

$$
\sigma(\zeta)=\bigcup_{i=1}^{n(\boldsymbol{\zeta})-1} \bigcup_{j=1}^{n_{i}(\boldsymbol{\zeta})} \tilde{\gamma}_{j}^{i}(\boldsymbol{\zeta})
$$

Lemma 4.1. Bound (3.83) holds for a quantum contour $\zeta$, with

$$
t=2 \log (2 d)+1 \text { and } p=\frac{\beta}{2 d}\left((\mu-1)-\frac{(m+1)}{4 d}-(2 \log (2 d)+1)(2 e d)^{4 d}(m+1)\right) .
$$

Proof. First, we estimate, from above, the factor $\prod_{i=0}^{n(\boldsymbol{\zeta})-1} X^{i}\left(y_{i}^{-}\right)$in (2.15). Given a quantum contour $\boldsymbol{\zeta}$, fix a sequence $0 \leq i_{1}<\ldots<i_{k} \leq n(\boldsymbol{\zeta})$ of the indices of the classical configurations $X^{i}=X^{i}(\mathbf{X}(\zeta))$ such that $X^{i_{j}}\left(y_{i_{j}}^{-}\right) \geq \frac{m}{2}+1$. The sites $y_{i_{j}}^{-}$may be partitioned into two groups. The first group consists of the sites with $X^{i_{j}}\left(y_{i_{j}}^{-}\right)=X^{0}\left(y_{i_{j}}^{-}\right)$. The second one consists of the sites with $X^{i_{j}}\left(y_{i_{j}}^{-}\right) \neq X^{0}\left(y_{i_{j}}^{-}\right)$. The cyclic property of $\zeta$ implies that for every site $y_{i_{j}}^{-}$from the first group, there exists a conjugate site, $y_{i_{j}^{\prime}}^{-}$, from $\left\{y_{1}^{-}, \ldots, y_{n(\boldsymbol{\zeta})}^{-}\right\} \backslash\left\{y_{i_{1}}^{-}, \ldots, y_{i_{k}}^{-}\right\}$, such that $i_{j}^{\prime}>i_{j}, y_{i_{j}}^{-}=y_{i_{j}^{\prime}}^{+}$ and

$$
X^{i_{j}^{\prime}}\left(y_{i_{j}}^{-}\right)=X^{i_{j}}\left(y_{i_{j}}^{-}\right)-1 \text {. }
$$

Clearly,

$$
X^{i_{j}^{\prime}}\left(y_{i_{j}^{\prime}}^{+}\right)+X^{i_{j}^{\prime}}\left(y_{i_{j}^{\prime}}^{-}\right) \leq m
$$


and

$$
X^{i_{j}}\left(y_{i_{j}}^{-}\right)+X^{i_{j}^{\prime}}\left(y_{i_{j}^{\prime}}^{-}\right) \leq m+1
$$

Similarly, for every site $y_{i_{j}}^{-}$from the second group, there exists a conjugate site, $y_{i_{j}^{\prime}}^{-}$, from $\left\{y_{1}^{-}, \ldots, y_{n(\boldsymbol{\zeta})}^{-}\right\} \backslash\left\{y_{i_{1}}^{-}, \ldots, y_{i_{k}}^{-}\right\}$, such that $i_{j}^{\prime}<i_{j}, y_{i_{j}}^{-}=y_{i_{j}^{\prime}}^{+}$and

$$
X^{i_{j}^{\prime}}\left(y_{i_{j}}^{-}\right)=X^{i_{j}}\left(y_{i_{j}}^{-}\right)-1
$$

which yields

$$
X^{i_{j}}\left(y_{i_{j}}^{-}\right)+X^{i_{j}^{\prime}}\left(y_{i_{j}^{\prime}}^{-}\right) \leq m+1
$$

By construction, we have $X^{i}\left(y_{i}^{-}\right)<\frac{m}{2}$ for any site $y_{i}$ from $\left\{y_{1}^{-}, \ldots, y_{n(\boldsymbol{\zeta})}^{-}\right\} \backslash$ $\left\{y_{i_{1}}^{-}, \ldots, y_{i_{k}}^{-}\right\}$. Obviously

$$
\begin{aligned}
& \max _{g=} \quad(g h)=\frac{(m+1)(m+1)}{4} .2,+1, \ldots, m \\
& h=1, \ldots, m \\
& g+h \leq m+1
\end{aligned}
$$

This implies the bound

$$
\prod_{i=0}^{n(\zeta)-1} X^{i}\left(y_{i}^{-}\right) \leq\left(\frac{(m+1)(m+1)}{4}\right)^{n(\boldsymbol{\zeta}) / 2}
$$

Now assume that

$$
n(\zeta) \geq \frac{1}{2 d}(2 e d)^{4 d} \beta(m+1)
$$

Then

$$
\begin{aligned}
\left(\frac{\beta}{2 d}\right)^{n(\boldsymbol{\zeta})} \frac{1}{n(\boldsymbol{\zeta}) !} \prod_{i=0}^{n(\boldsymbol{\zeta})-1} X^{i}\left(y_{i}^{-}\right) & \leq\left(\frac{\beta(m+1)}{4 d}\right)^{n(\boldsymbol{\zeta})} \frac{1}{n(\boldsymbol{\zeta}) !} \\
& \leq\left(\frac{\beta e(m+1)}{4 d n(\boldsymbol{\zeta})}\right)^{n(\boldsymbol{\zeta})} \\
& \leq\left(\frac{e}{2(2 e d)^{4 d}}\right)^{n(\boldsymbol{\zeta})} .
\end{aligned}
$$

Observe that

$$
|\sigma(\zeta)| \leq 2 d n(\zeta)
$$

because of the bound

$$
\left|\bigcup_{j=1}^{n_{i}(\boldsymbol{\zeta})} \tilde{\boldsymbol{\gamma}}_{j}^{i}(\boldsymbol{\zeta})\right|+2 d \geq\left|\bigcup_{j=1}^{n_{i+1}(\boldsymbol{\zeta})} \tilde{\boldsymbol{\gamma}}_{j}^{i+1}(\boldsymbol{\zeta})\right|
$$


In turn, bound (4.13) means that, while passing from classical configuration $X^{i}(\boldsymbol{\zeta})$ to $X^{i+1}(\zeta)$, which corresponds to a single jump, the number of plaquettes in the interface cannot increase more than by $2 d$. Bounds (4.11) and (4.12) together give

$$
\begin{aligned}
\left(\frac{\beta}{2 d}\right)^{n(\boldsymbol{\zeta})} \frac{1}{n(\boldsymbol{\zeta}) !} \prod_{i=0}^{n(\boldsymbol{\zeta})-1} X^{i}\left(y_{i}^{-}\right) & \leq\left(\frac{e}{2(2 e d)^{4 d}}\right)^{|\sigma(\boldsymbol{\zeta})| /(2 d)} \\
& \leq \exp (-t|\sigma(\boldsymbol{\zeta})|)
\end{aligned}
$$

Hence, under condition (4.10),

$$
w(\zeta) \leq \exp \left(-\beta(\mu-1) \frac{1}{2 d}\left\|\left\{\boldsymbol{\omega}_{j}(\zeta)\right\}\right\|-t|\sigma(\zeta)|\right)
$$

On the other hand, if

$$
n(\zeta)<\frac{1}{2 d}(2 e d)^{4 d} \beta(m+1)
$$

then

$$
|\sigma(\zeta)|<(2 e d)^{4 d} \beta(m+1)
$$

Therefore,

$$
\begin{aligned}
w(\boldsymbol{\zeta}) \leq & \exp \left(-\beta(\mu-1) \frac{1}{2 d}\left\|\left\{\boldsymbol{\omega}_{j}(\boldsymbol{\zeta})\right\}\right\|+\frac{\beta(m+1)}{4 d}\right) \\
\leq & \exp \left[-\beta\left((\mu-1) \frac{1}{2 d}\left\|\left\{\boldsymbol{\omega}_{j}(\boldsymbol{\zeta})\right\}\right\|-\frac{m+1}{4 d}\right.\right. \\
& \left.\left.-t(2 e d)^{4 d}(m+1)\right)-t|\sigma(\zeta)|\right] \\
\leq & \exp \left[-\beta\left((\mu-1)-\frac{m+1}{4 d}-t(2 e d)^{4 d}(m+1)\right)\right. \\
& \left.\times \frac{1}{2 d}\left\|\left\{\boldsymbol{\omega}_{j}(\boldsymbol{\zeta})\right\}\right\|-t|\sigma(\boldsymbol{\zeta})|\right]
\end{aligned}
$$

because $\left\|\left\{\boldsymbol{\omega}_{j}(\boldsymbol{\zeta})\right\}\right\| \geq 2 d$. Bounds (4.15) and (4.18) immediately give the lemma.

We now have to choose values of $u$ and $v$ figuring in Propositions 3.8 and 3.10. Namely, set $u=4$ and $v=e \beta m$. Recall that in Section 3 the choice of $u$ and $v$ specifies the long and short contours. In this section we apply the general scheme from Section 3 to a particular situation of the quantum contours. Following Section 3, we use, for a quantum contour, the notation $\varepsilon$ and $\zeta$, depending on the category into which it falls. Namely, we call a quantum contour $\varepsilon$ short if

$$
\operatorname{diam} \widetilde{\omega}_{i}(\varepsilon)<u
$$

cf. (3.82). The quantum contours for which condition (4.19) does not hold are called long and denoted by $\zeta$. 
The restricted partition functions $Z^{(l)}(V)$ are defined as

$$
Z^{(l)}(V)=\sum_{\left\{\varepsilon_{i}\right\}:\left\{\widetilde{\varepsilon}_{i}\right\} \subseteq V, E\left(\boldsymbol{\omega}_{j}\left(\varepsilon_{i}\right)\right)=l} \prod_{i} w\left(\varepsilon_{i}\right),
$$

cf. (3.61).

Lemma 4.2. There exist positive constants, $\bar{\mu}=\bar{\mu}(d)$ and $\bar{\beta}=\bar{\beta}(d)$ such that, for $u=4, v=e \beta m$ and $p$ and $t$ as in Lemma 4.1, and $\mu \geq \bar{\mu} m, \beta \geq \bar{\beta} m$, the following bounds hold:

(i) for $m$ even

$$
\begin{aligned}
\frac{Z^{(l)}(V)}{Z^{(m / 2)}(V)} & \leq \exp \left[-\left|[V]_{v}\right| 8 d \exp (-(p-2 \log (2 d)-2)(d u-u+1))\right], \\
l & =0, \ldots, m, \quad l \neq \frac{m}{2}
\end{aligned}
$$

(ii) for $m$ odd

$$
\begin{gathered}
\frac{Z^{(l)}(V)}{Z^{((m \pm 1) / 2)}(V)} \leq \exp \left[-\left|[V]_{v}\right| 8 d \exp (-(p-2 \log (2 d)-2)(d u-u+1))\right] \\
l=0, \ldots, m, \quad l \neq \frac{m}{2} \pm \frac{1}{2} .
\end{gathered}
$$

Proof. We discuss in detail the case of $m$ even: the case of $m$ odd is covered by a similar argument. We use Theorem 3.1 to evaluate the difference

$$
b=\log Z^{(l)}(V)-\log Z^{(m / 2)}(V) .
$$

Condition (3.2) figuring in Theorem 3.1 is valid, with $a(\varepsilon)=\left\|\left\{\widetilde{\omega}_{j}(\varepsilon)\right\}\right\|+|\sigma(\varepsilon)|$, by virtue of Lemma 4.1 .

In view of the restriction $\operatorname{diam} \widetilde{\omega}_{j}(\varepsilon) \leq 3$ (see (4.19)), it is easy to list all possible shapes of $\tilde{\omega}_{j}(\varepsilon)$. It can be: either

(i) the collection of $2 d$ plaquettes forming the surface of a unit cube on $\widetilde{\mathbb{Z}}^{d}$ centered at $x \in \mathbb{Z}^{d}$ (we denote such an interface by $\vartheta_{0}(x)$ );

or

(ii) the collection of $2 d(2 d-1)$ plaquettes forming a boundary of a subset of $\mathbb{Z}^{d}$ which consists of some site $x \in \mathbb{Z}^{d}$ united with all its nearest-neighbor sites (we denote such an interface by $\vartheta_{1}(x)$ ).

In the case where the reference to a particular site $x$ is not important, we write simply $\vartheta_{0}$ instead of $\vartheta_{0}(x)$ and $\vartheta_{1}$ instead of $\vartheta_{1}(x)$. Observe that the partition function inside the volume enclosed by $\vartheta_{1}$ is not trivial (it contains terms corresponding to collections of the type one interfaces). Therefore, to satisfy the compatibility rule for the short quantum contours, we have to modify the statistical weight of the short quantum contour $\varepsilon$ with $\widetilde{\omega}(\varepsilon)=\vartheta_{1}$, by multiplying it by a ratio of the conditioned partition functions

$$
\frac{Z^{(I(\boldsymbol{\omega}(\boldsymbol{\varepsilon})))}(\operatorname{Int} \widetilde{\omega}(\varepsilon) \mid \varepsilon)}{Z^{(E(\boldsymbol{\omega}(\varepsilon)))}\left(\operatorname{Int} \widetilde{\omega}(\varepsilon) \mid \varepsilon^{*}\right)}
$$


which are defined as in (3.67), with replacing $\boldsymbol{\omega}_{j}\left(\Omega_{n}\right)$ by $\varepsilon$. It is easy to see that ratio (4.24) is less than 2 .

To estimate $b$, we begin with analysing the contribution to $\ln Z^{(l)}(V)$ which comes from polymers $\pi$ consisting of a single short quantum contour $\varepsilon$, with the set of bold plaquettes $\tilde{\omega}(\varepsilon)$ coinciding with $\vartheta_{0}(x)$, and with $|E(\omega(\varepsilon))-I(\omega(\varepsilon))|=1$. The statistical weight of such a polymer coincides with the statistical weight of $\varepsilon$ and is

$$
w(\pi)=w(\varepsilon)=\exp (-\beta(\mu-1))\left(\frac{\beta}{2 d}\right)^{n(\varepsilon)} \frac{1}{(n(\varepsilon)) !}(l \cdot(m-l))^{n(\varepsilon) / 2}
$$

Obviously, the right-hand side of (4.25) is maximal for $l=\frac{m}{2}$. Observe now that there exists exactly $(2 d)^{n(\varepsilon)}$ different short quantum contours $\varepsilon$, with $\widetilde{\omega}(\varepsilon)=\vartheta_{0}(x)$ and $|E(\boldsymbol{\omega}(\varepsilon))-I(\boldsymbol{\omega}(\varepsilon))|=1$ and fixed $n(\varepsilon)$. [These quantum contours correspond to $n(\varepsilon)$ independent jumps in any of $2 d$ directions.] Hence, the contribution to $b$ coming from the polymers just described is

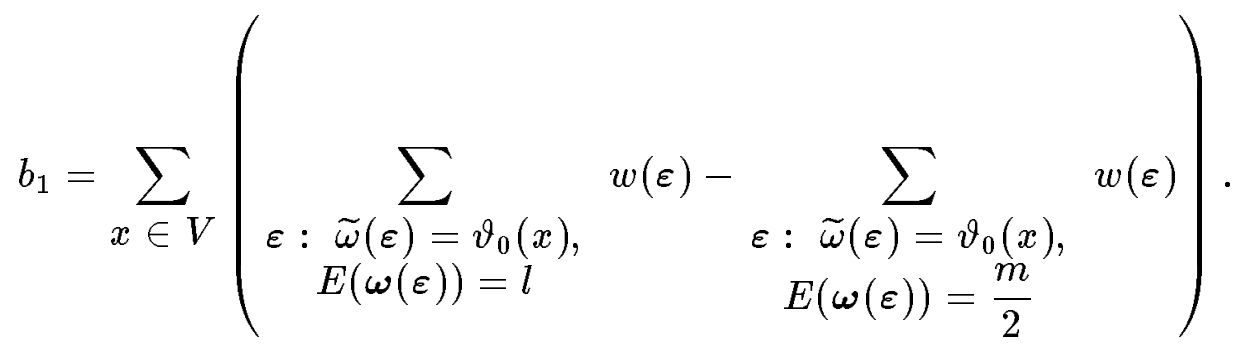

It is not hard to see that

$$
\begin{aligned}
& b_{1} \leq\mid V]_{v} \mid \sum_{n=1}^{v} \exp (-\beta(\mu-1))\left(\frac{\beta}{2 d}\right)^{n} \frac{(2 d)^{n}}{n !} \\
& \times\left((l \cdot(m-l))^{n / 2}-\left(\frac{m}{2} \cdot \frac{m}{2}\right)^{n / 2}\right) \\
& \leq\mid V]_{v} \mid \exp (-\beta(\mu-1))\left(\exp (\beta \sqrt{l \cdot(m-l)})-\exp \left(\beta \frac{m}{2}\right)\right) \\
& \leq-\left|[V]_{v}\right| \exp \left(-\beta\left(\mu-1-\frac{m}{2}\right)\right)(1-\exp (-\beta / m)) .
\end{aligned}
$$

Second, we take into account the contribution to $b$ which comes from polymers $\pi$ consisting of a single short quantum contour $\varepsilon$, with the set of bold plaquettes $\widetilde{\omega}(\varepsilon)$ coinciding with $\vartheta_{0}(x)$, and with $|E(\boldsymbol{\omega}(\varepsilon))-I(\boldsymbol{\omega}(\varepsilon))| \geq 2$. As before, the statistical weight of such a polymer coincides with the statistical weight of $\varepsilon$ and obeys

$$
\begin{aligned}
& w(\pi)=w(\varepsilon) \leq \exp (-\beta(\mu-1) \cdot|E(\boldsymbol{\omega}(\varepsilon))-I(\boldsymbol{\omega}(\varepsilon))|) \\
& \left(\frac{\beta}{2 d}\right)^{n(\varepsilon)} \frac{1}{(n(\varepsilon)) !}\left(\frac{m+1}{2}\right)^{n(\varepsilon)},
\end{aligned}
$$

as follows from (2.15) and (4.9). Observe that the number of different short quantum contours $\varepsilon$, with $\widetilde{\omega}(\varepsilon)=\vartheta_{0}(x)$ and with fixed $n(\varepsilon)$ does not exceed

$$
(2 d|E(\omega(\varepsilon))-I(\boldsymbol{\omega}(\varepsilon))|)^{n(\varepsilon)} .
$$


Hence, the contribution to $b$ coming from the polymers of this type is

$$
\begin{aligned}
b_{2} & \leq 2\left|[V]_{v}\right| \sum_{n=1}^{\infty} 2 \sum_{k=2}^{m} \exp (-\beta(\mu-1) k)\left(\frac{\beta}{2 d}\right)^{n} \frac{(2 d k)^{n}}{n !}\left(\frac{m+1}{2}\right)^{n} \\
& \leq 4\left|[V]_{v}\right| \sum_{k=2}^{m} \exp \left(-\beta\left(\mu-1-\frac{m+1}{2}\right) k\right) \\
& \leq 8\left|[V]_{v}\right| \exp \left(-\beta\left(\mu-1-\frac{m+1}{2}\right) 2\right)
\end{aligned}
$$

the last inequality holds for $\exp \left[-\beta\left(\mu-1-\frac{m+1}{2}\right) 2\right]<\frac{1}{2}$.

The next contribution to $b$ to be assessed comes from polymers $\pi$ consisting of a single short quantum contour $\varepsilon$ described in (ii). The statistical weight of such a polymer coincides with the modified statistical weight of $\varepsilon$ and obeys

$$
\begin{aligned}
& w(\pi)=2 w(\varepsilon) \leq 2 \exp [-\beta(\mu-1)(2 d-1) \cdot|E(\boldsymbol{\omega}(\varepsilon))-I(\boldsymbol{\omega}(\varepsilon))|] \\
& \times\left(\frac{\beta}{2 d}\right)^{n(\varepsilon)} \frac{1}{(n(\varepsilon)) !}\left(\frac{m+1}{2}\right)^{n(\varepsilon)} .
\end{aligned}
$$

Bound (4.30) follows from (2.15), (4.9) and the equality $\left|\vartheta_{1}(x)\right|=2 d(2 d-1)$. Observe that the number of different short quantum contours $\varepsilon$, with $\widetilde{\omega}(\varepsilon)=\vartheta_{1}(x)$ and fixed $n(\varepsilon)$, does not exceed $(2 d 2 d|E(\omega(\varepsilon))-I(\boldsymbol{\omega}(\varepsilon))|)^{n(\varepsilon)}$. Hence, the contribution to $b$ coming from polymers of this type is

$$
\begin{aligned}
& b_{3} \leq 2 \cdot 2\left|[V]_{v}\right| \sum_{n=1}^{\infty} 2 \sum_{k=1}^{m} \exp (-\beta(\mu-1)(2 d-1) k) \\
& \times\left(\frac{\beta}{2 d}\right)^{n} \frac{(2 d 2 d k)^{n}}{n !}\left(\frac{m+1}{2}\right)^{n} \\
& \leq 8\left|[V]_{v}\right| \sum_{k=1}^{m} \exp \left[-\beta\left(\mu-1-\frac{m+1}{2}\right)(2 d-1) k+\beta \frac{m+1}{2} k\right] \\
& \leq 16\left|[V]_{v}\right| \exp \left[-\beta\left(\mu-1-\frac{m+1}{2}\right)(2 d-1)+\beta \frac{m+1}{2}\right] ;
\end{aligned}
$$

the last inequality holds for $\exp \left[-\beta\left(\mu-1-\frac{m+1}{2}\right)(2 d-1)+\beta \frac{m+1}{2}\right]<\frac{1}{2}$.

Finally, consider the contribution to $b$ which comes from polymers $\pi=\left[\varepsilon_{j}^{\alpha_{j}}\right]$ containing at least two short quantum contours. Here, we use bound (3.7). More precisely, denoting by $\left[\varepsilon^{\prime}, \varepsilon^{\prime \prime}\right]$ the polymer contaning a pair of distinct (incompatible) short quantum contours $\varepsilon^{\prime}, \varepsilon^{\prime \prime}$, with multiplicity one, this contribution is

$$
b_{4} \leq \sum_{\substack{\varepsilon^{\prime}, \varepsilon^{\prime \prime}: \varepsilon^{\prime} \text { and } \varepsilon^{\prime \prime} \\ \text { incompatible }}} \sum_{\pi:\left[\varepsilon^{\prime}, \varepsilon^{\prime \prime}\right]} w(\pi)
$$




$$
\leq \sum_{\substack{\varepsilon^{\prime}, \varepsilon^{\prime \prime}: \varepsilon^{\prime} \text { and } \varepsilon^{\prime \prime} \\ \text { incompatible }}} w\left(\varepsilon^{\prime}\right) w\left(\varepsilon^{\prime \prime}\right) \exp \left(a\left(\varepsilon^{\prime}\right)+a\left(\varepsilon^{\prime \prime}\right)\right) .
$$

The last sum does not exceed

$$
\begin{aligned}
& \sum_{n^{\prime}=0}^{\infty} \sum_{n^{\prime \prime}=0}^{\infty} \sum_{\substack{x^{\prime}, x^{\prime \prime} \in V: \\
\operatorname{dist}\left(x^{\prime}, x^{\prime \prime}\right) \leq n^{\prime}+n^{\prime \prime}}} \sum_{\substack{\tilde{\omega}\left(\varepsilon^{\prime}\right)=\vartheta_{0}\left(x^{\prime}\right), n\left(\varepsilon^{\prime}\right)=n^{\prime}}} w\left(\varepsilon^{\prime}\right) \exp \left(a\left(\varepsilon^{\prime}\right)\right) \\
& +\sum_{\substack{\varepsilon^{\prime}: \widetilde{\omega}\left(\varepsilon^{\prime}\right)=\vartheta_{1}\left(x^{\prime}\right), n\left(\varepsilon^{\prime}\right)=n^{\prime}}} w\left(\varepsilon^{\prime}\right) \exp \left(a\left(\varepsilon^{\prime}\right)\right) \\
& \times\left(\sum_{\varepsilon^{\prime \prime}: \widetilde{\omega}\left(\varepsilon^{\prime \prime}\right)=\vartheta_{0}\left(x^{\prime \prime}\right)} w\left(\varepsilon^{\prime \prime}\right) \exp \left(a\left(\varepsilon^{\prime \prime}\right)\right)\right. \\
& \left.+\sum_{\varepsilon^{\prime \prime}: \begin{array}{c}
\widetilde{\omega}\left(\varepsilon^{\prime \prime}\right)=\vartheta_{1}\left(x^{\prime \prime}\right), \\
n\left(\varepsilon^{\prime \prime}\right)=n^{\prime \prime}
\end{array}} w\left(\varepsilon^{\prime \prime}\right) \exp \left(a\left(\varepsilon^{\prime \prime}\right)\right)\right) .
\end{aligned}
$$

The number of pairs $x^{\prime}, x^{\prime \prime} \in V$ with dist $\left(x^{\prime}, x^{\prime \prime}\right) \leq n^{\prime}+n^{\prime \prime}$ is $\leq\left(n^{\prime}+n^{\prime \prime}\right)^{d} \leq$ $\exp \left(d\left(n^{\prime}+n^{\prime \prime}\right)\right)$. Consequently, the right-hand side of (4.33) is

$$
\begin{aligned}
& \leq|V| \sum_{n=0}^{\infty} \exp (2 d n)\left(\sum_{\begin{array}{c}
\widetilde{\omega}(\varepsilon)=\vartheta_{0}(0), \\
n(\varepsilon)=n
\end{array}} w(\varepsilon) \exp (a(\varepsilon))\right. \\
& \left.+\sum_{\substack{\varepsilon: \widetilde{\omega}(\varepsilon)=\vartheta_{1}(0), n(\varepsilon)=n}} w(\varepsilon) \exp (a(\varepsilon))\right)^{2} .
\end{aligned}
$$

Arguing as before (see (4.12), (4.29) and (4.31)), we bound (4.34) by

$$
|V| \sum_{n=0}^{\infty} \exp (2 d n) \sum_{k=1}^{m}(2 \exp (-\beta(\mu-1) k+2 d k)
$$




$$
\begin{gathered}
\quad\left(\frac{\beta}{2 d}\right)^{n} \frac{(2 d k)^{n}}{n !}\left(\frac{m+1}{2}\right)^{n} \exp (2 d n) \\
+2 \exp (-\beta(\mu-1)(2 d-1) k+2 d(2 d-1) k) \\
\left.\times\left(\frac{\beta}{2 d}\right)^{n} \frac{(2 d 2 d k)^{n}}{n !}\left(\frac{m+1}{2}\right)^{n} \exp (2 d n)\right)^{2} \\
\leq 2|V| \sum_{k}^{m}\left(\exp \left(-\beta\left(\mu-1-\frac{m+1}{2}\right) k+2 d k+\exp (3 d)\right)\right. \\
+\exp \left(-\beta\left(\mu-1-\frac{m+1}{2}\right)(2 d-1) k\right. \\
\left.\left.+\beta \frac{m+1}{2} k+2 d(2 d-1) k+\exp (3 d)\right)\right)^{2} \\
\leq 8|V| \sum_{k}^{m}\left(\exp \left(-2 \beta\left(\mu-1-\frac{m+1}{2}\right) k+4 d k+2 \exp (3 d)\right)\right) \\
\leq 16|V|\left(\exp \left(-2 \beta\left(\mu-1-\frac{m+1}{2}\right)+4 d+2 \exp (3 d)\right)\right)
\end{gathered}
$$

the last three inequalities hold for $\exp \left(-2 \beta\left(\mu-1-\frac{m+1}{2}\right)\right)<\frac{1}{2}$.

For $\mu \geq 3 m$ and

$$
\beta \geq \max \left[3 m \log 2, \frac{6 \log 2+4 d+2 \exp (3 d)+\log v}{\mu-m-2}\right],
$$

we can combine (4.27), (4.29), (4.31) and (4.35) and obtain

$$
b \leq-\frac{1}{2}\left|[V]_{v}\right| \exp \left[-\beta\left(\mu-1-\frac{m}{2}\right)\right] .
$$

Now it is easy to see that, for

$$
\begin{aligned}
\bar{\mu} & =6(2 \log (2 d)+1)(2 e d)^{4 d}, \\
\bar{\beta} & =32 \exp (2 d), \\
\mu & \geq \bar{\mu} m \\
\beta & \geq \bar{\beta} m, \\
u & =4 \\
v & =e \beta m, \\
p & =\frac{\beta}{2 d}\left[(\mu-1)-\frac{(m+1)}{4 d}-(2 \log (2 d)+1)(2 e d)^{4 d}(m+1)\right],
\end{aligned}
$$

we have

$$
(p-2 \log (2 d)-2)(d u-u+1) \geq \beta\left(\mu-1-\frac{m}{2}\right)+\log (8 d),
$$

which gives the lemma.

Note that the value $\bar{\mu}(d)$ is of order $d^{d}$ and not an optimal one. In fact, our method allows us to reduce $\bar{\mu}(d)$, after a tedious calculation, to a quantity of order $d$. 
As was noted before, Lemma 4.2 allows us to prove, by means of a standard argument, the existence of the limiting states $\phi^{0}$ and $\phi^{ \pm}$. More precisely, it implies the existence of the limits (1.16), for $Y=Y^{(m / 2)}$ in the case of $m$ even and for $Y=Y^{(m / 2 \pm 1 / 2)}$ in the case of $m$ odd; in the last case it also implies that the limiting reduced density matrices $\pi_{V^{0}}^{ \pm}$are distinct. The translation-invariance, periodicity and symmetry properties of the families of the limiting reduced density matrices are valid by construction. The correlation-decay property needed for ergodicity is established in a standard way, on the base of the polymer expansions obtained.

To complete the proof of Theorem 1 , we have to establish the uniqueness of states $\phi^{0}$ and $\phi^{ \pm}$, in the sense stated in Theorem 1. For this purpose, consider a cube $V$ on $\mathbb{Z}^{d}$, with boundary condition $Y_{V^{c}}$, where $Y$ is an arbitrary translation-periodic configuration. As in the previously discussed case of $Y=Y^{(l)}$, we can define classical contours and quantum contours under boundary condition $Y$. The difference with the previous case is that now the classical (and hence quantum) contours that 'touch' the boundary $\partial V$ may be 'open', in the sense that the interface of such contours, still being connected sets of plaquettes of $\widetilde{\mathbb{Z}}^{d}$, do not neccessarily enclose a subset of $V$.

Let $\tilde{Y}_{V}$ be a configuration in $V$ such that

(i) $\tilde{Y}_{V}+Y_{V^{c}}$ is admissible,

(ii) $\tilde{Y}_{[V]_{m}}=Y_{[V]_{m}}^{(m / 2)}$ for $m$ even and $\tilde{Y}_{[V]_{m}}=Y_{[V]_{m}}^{((m \pm 1) / 2)}$ for $m$ odd,

(iii) the compatible collection of classical contours $\left\{\boldsymbol{\gamma}_{i}\right\}$ corresponding to $\tilde{Y}_{V}$ has a minimal value of $\left\|\left\{\gamma_{i}\right\}\right\|$ (in the class of the configuration in $V$ which satisfy conditions (i), (ii)).

It is not hard to check that such $\tilde{Y}_{V}$ exists, and $\left\|\left\{\gamma_{i}\left(\tilde{Y}_{V}\right)\right\}\right\| \leq m|\partial V|$. Using, in (2.5) and (2.6), $\tilde{Y}_{V}$ instead of $Y_{V}$, it is possible to repeat the previous construction and define bulky contours $\widetilde{\Omega}$ similar to those figuring in Propositions 3.8 and 3.10. These bulky contours have the same statistical weight as before (which satisfies bound (3.75)), provided that they do not touch $\partial V$. Otherwise, their statistical weight satisfies a weaker bound

$$
\begin{aligned}
|w(\tilde{\Omega})| \leq \exp ( & -\sum_{n}\left((p-1)||\left\{\omega_{j}\left(\zeta_{n}(\widetilde{\Omega})\right)\right\}||+t\left|\sigma\left(\widetilde{\zeta}_{n}(\widetilde{\Omega})\right)\right|\right)-q|\widetilde{\Omega}| \\
& \left.+\beta(\mu-1) \frac{m}{2 d}|\partial V \cap \partial \widetilde{\Omega}|+|\partial V \cap \partial \widetilde{\Omega}|\right)
\end{aligned}
$$

Comparing with (3.75), there are two new summands here, $\beta(\mu-1) \frac{m}{2 d}|\partial V \cap \partial \widetilde{\Omega}|$ and $|\partial V \cap \partial \widetilde{\Omega}|$. The first comes from term $\beta(\mu-1)\left|\widetilde{Y}_{V}\right|$ in (2.5) and (2.6). The second arises because, instead of (3.86), we now have, for the $\widetilde{\Omega}$ 's under consideration, a bound

$$
\frac{Z^{(k)}(\tilde{\Omega})}{Z^{(0)}(\tilde{\Omega})} \leq \exp \left(-\left|[\widetilde{\Omega}]_{v}\right| 8 d \exp (-(p-2 \log (2 d)-2)(d u-u+1))+|\partial V \cap \partial \tilde{\Omega}|\right)
$$

In turn, the term $|\partial V \cap \partial \widetilde{\Omega}|$ appears in (4.38) and (4.37) in order to compensate the contribution coming from the short quantum contours in $\widetilde{\Omega}$ touching $\partial V \cap \partial \widetilde{\Omega}$. [These short quantum contours have a modified statistical weight which we do not write in an exact form.] 
Bound (4.39) implies that, for any $\tilde{\Omega}$ that touches $\partial V$ and has $|\widetilde{\Omega}| \geq$ $|V|^{1-1 / d+\delta}$ and $0<\delta<\frac{1}{d}$, the probability of having such $\tilde{\Omega}$, in the ensemble with boundary condition $Y_{V^{c}}$, tends to zero, with $V \nearrow \mathbb{Z}^{d}$. This observation implies, by means of a standard argument (see, e.g. [ $\mathrm{Z}]$ ), the uniqueness of the limiting states $\phi^{0}$ and $\phi^{ \pm}$(in the class of possible ergodic limiting states, with the boundary conditions given by the translation-periodic classical configurations). Hence, any limiting state (1.11) (actually, any limit point) coincides with $\phi^{0}$ for $m$ even and is a mixture of $\phi^{ \pm}$for $m$ odd.

Finally, using the same standard argument, it is not hard to check that for $m$ odd and $Y=Y^{(l)}$, with $l=0, \ldots, \frac{m-1}{2} \quad\left(l=\frac{m+1}{2} \ldots, m\right)$, the configuration $\tilde{Y}_{V}=Y_{V}^{(m / 2-1 / 2)}$ (respectively, $\left.\tilde{Y}_{V}=Y_{V}^{(m / 2+1 / 2)}\right)$. This gives relations $(1.12 \mathrm{a}, \mathrm{b})$ and completes the proof of Theorem 1.

Relations (1.18), (1.19) which in turn imply Theorem 2 follow immediately from the fact that the probability of having a quantum contour $\zeta$ with Int $\widetilde{\omega}_{j}(\zeta) \ni 0$ tends to 0 as $\beta \rightarrow \infty$.

Acknowledgements. A.E.M. thanks St John's College, Cambridge, for its warm hospitality and support. Both authors thank the Isaac Newton Institute, University of Cambridge, for its warm hospitality during the Random Spatial Processes program. Y.M.S. thanks the Institute for Mathematics and its Applications, University of Minnesota, for support towards this work. 


\section{References}

1. [ BR ] O. Bratteli and D.W. Robinson, Operator Algebras and Quantum Statistical Mechanics, vol 1, Berlin: Springer-Verlag, 1979; vol. 2, Berlin: SpringerVerlag, 1981

2. [ DMaSin ] E.A. Dinaburg, A.E. Mazel and Ya.G. Sinai, The ANNNI model and contour models with interaction, in: Mathematical Physics Reviews $C$, vol. 6 (S.P. Nolvikov, ed.), New York: Gordon and Breach, 1987, pp. 113-168

3. [ DyLSi ] F. Dyson, E. Lieb and B. Simon, Phase transitions in quantum spin systems with isotropic and anisotropic interactions. Journ. Stat. Phys. 18 (1978), $335-383$

4. [ FSiSp ] J. Fröhlich, B. Simon and T. Spencer, Infrared bounds, phase transitions and continuous symmetry breaking. Commun. Math. Phys. 50 (1976), $79-85$

5. [ G1 ] J. Ginibre, Existence of phase transitions for quantum lattice systems. Commun. Math. Phys. 14 (1969), 205-234

6. [ G2 ] J. Ginibre, Some applications of functional integration in statistical mechanics, in: Statistical Mechanics and Field Theory (C. de Witt and R. Stora, Eds), New York: Gordon and Breach, 1970, 327-428

7. [ KL ] T. Kennedy and E.H. Lieb, An itinerant electron model with crystalline or magnetic long range order. Physica A 138 (1986), 320-358

8. [ KoPr] R. Kotecky and D. Preiss, Cluster expansion for abstract polymer models Commun. Math. Phys. 103 (1986), 491-498

9. [ M1 ] V.A. Malyshev, Cluster expansions in lattice models of statistical physics and the quantum theory of fields. Russian Math. Surveys 35 (1980), 3-53

10. [ M2 ] V.A. Malyshev, Soliton sectors in lattice models with continuous time. Funkts. Anal. Prilozhen. [Russian] 13, no. 1 (1979), 31-41

11. [ MMi] V.A. Malyshev and R.A. Minlos, Gibbs Random Fields. The method of Cluster Expansions [Russian], Moscow: Nauka, 1985

12. [ MaSu ] A.E. Mazel and Yu.M. Suhov, Random surfaces with two-sided constarints: an application of the theory of dominant ground states. J. Stat. Phys. 64, no. $1 / 2$ (1991), 111-134

13. [ MeMir-So ] A. Messager and S. Miracle-Sole, in preparation

14. [ OPiSu ] E. Olivieri, P. Picco and Yu.M. Suhov, On the Gibbs states for one-dimensional lattice boson systems with a long-range ineraction. J. Stat. Phys. 70, no $3 / 4$ (1993), 985-1028

15. [ P ] Y.M. Park, Quantum statistical mechanics for superstable interactions: Bose - Einstein statistics. J. Stat. Phys. 40 (1985), 259-302

16. [ R ] D.W. Robinson, A proof of the existence of the phase transition in the anisotropic Heisenberg model. Commun. Math. Phys. 14 (1969), 195-204 
17. [ S ] E. Seiler, Gauge theories as a problem of constructive quantum field theory and statistical mechanics. Lect. Notes in Physics, vol. 159, Berlin: SpringerVerlag, 1982

18. [ Su ] Yu.M. Suhov, Limit Gibbs state for a class of one-dimensional systems of quantum statistical mechanics. Commun. Math. Phys., 62 (1978), 119-136

19. [ Z ] M. Zahradnik, An alternative version of Pirogov - Sinai theory. Commun. Math. Phys. 93 (1984), 559-581 